\title{
Concurrent Imitation Dynamics in Congestion Games*
}

\author{
Heiner Ackermann \\ RWTH Aachen University \\ ackermann@cs.rwth-aachen.de \\ Simon Fischer \\ RWTH Aachen University \\ fischer@cs.rwth-aachen.de
}

\author{
Petra Berenbrink ${ }^{\dagger}$ \\ Simon Fraser University \\ petra@cs.sfu.ca \\ Martin Hoefer ${ }^{\ddagger}$ \\ Stanford University \\ mhoefer@cs.rwth-aachen.de
}

October 23, 2018

\begin{abstract}
Imitating successful behavior is a natural and frequently applied approach to trust in when facing scenarios for which we have little or no experience upon which we can base our decision. In this paper, we consider such behavior in atomic congestion games. We propose to study concurrent imitation dynamics that emerge when each player samples another player and possibly imitates this agents' strategy if the anticipated latency gain is sufficiently large. Our main focus is on convergence properties. Using a potential function argument, we show that our dynamics converge in a monotonic fashion to stable states. In such a state none of the players can improve its latency by imitating somebody else.

As our main result, we show rapid convergence to approximate equilibria. At an approximate equilibrium only a small fraction of agents sustains a latency significantly above or below average. In particular, imitation dynamics behave like fully polynomial time approximation schemes (FPTAS). Fixing all other parameters, the convergence time depends only in a logarithmic fashion on the number of agents.

Since imitation processes are not innovative they cannot discover unused strategies. Furthermore, strategies may become extinct with non-zero probability. For the case of singleton games, we show that the probability of this event occurring is negligible. Additionally, we prove that the social cost of a stable state reached by our dynamics is not much worse than an optimal state in singleton congestion games with linear latency function. Finally, we discuss how the protocol can be extended such that, in the long run, dynamics converge to a Nash equilibrium.
\end{abstract}

\section{Introduction}

We study imitation dynamics that emerge if myopic players concurrently imitate each other in order to improve on their own situation. In scenarios for which players have little or no experience upon which they can base their decisions, or in which precise knowledge about the available options and their consequences is absent, it is a good strategy to imitate successful behavior. Thus, it is not surprising that such imitating behavior can frequently be observed, and has already been studied intensively in economics and game theory [20, 27].

We analyze such imitation dynamics in the context of symmetric congestion games [24]. As an example of such a game consider a network congestion game in which players strive to allocate paths with minimum latency between the same source-sink pair in a network. The latency of a path equals the sum of the latencies of the edges in that path and the latency of an edge depends on the number of players sharing it.

\footnotetext{
*This work was in part supported by the DFG through German UMIC-excellence cluster at RWTH Aachen University.

${ }^{\dagger}$ Supported by an NSERC grant. Part of this work was done while author visited RWTH Aachen University.

$\ddagger$ Supported by the German Academic Exchange Service (DAAD) within the PostDoc-Program.
} 
We consider a simple imitation rule according to which players strive to improve their individual latencies over time by imitating others in a concurrent and round-based fashion. This IMITATION PROTOCOL has several appealing properties: it is simple, stateless, based on local information, and is compatible with the selfish incentives of the players. The IMITATION PROTOCOL consists of a sampling and a migration step. First, each player samples another player uniformly at random. Then he considers the latency gain that he would have by adopting the strategy of the sampled player, under the assumption that no-one else changes his strategy. If this latency gain is not too small our player adopts the sampled strategy with a migration probability mainly depending on the anticipated latency gain. The major technical challenge in designing such a concurrent protocol is to avoid overshooting effects. Overshooting occurs if too many players sample other players currently using the same strategy, and if all of them migrate towards it. In this case their latency might be greater than before the migration. In order to avoid overshooting, the migration probabilities have to be defined appropriately without sacrificing the benefit of concurrency. We propose to scale the migration probabilities by the elasticity of the latency functions in order to avoid overshooting. The elasticity of a function at point $x$ describes the proportional growth of the function value as a result of a proportional growth of its argument. Note that in case of polynomial latency functions with positive coefficients and maximum degree $d$ the elasticity is upper bounded by $d$.

A natural solution concept in this scenario is imitation-stability. A state is imitation-stable if no more improvements are possible based on the IMITATION PROTOCOL. We analyse convergence properties with respect to this solution concept.

\subsection{Our Results}

As our first result we prove that the IMITATION PROTOCOL succeeds in avoiding overshooting effects and converges in a monotonic fashion (Section 3). More precisely, we show that a well-known potential function (Rosenthal [24]) decreases on expectation as long as the system is not yet at an imitation-stable state. Thus, the potential is a super-martingale and eventually reaches a local minimum, corresponding to an imitationstable state. Hence, as a corollary, we see that an imitation-stable state is reached in pseudopolynomial time.

Our main result, presented in Section 4, however, is a much stronger bound on the time to reach approximate imitation-stable states. What is a natural definition of approximately stable states in our setting? By repeatedly sampling other agents, an agent gets to know the average latency of the system. It is approximately satisfied, if it does not sustain a latency much larger than the average. Hence, we say that a state is approximately stable if almost all agents are almost satisfied. More precisely, we consider states in which at most a $\delta$-fraction of the agents deviates by more that an $\epsilon$-fraction (in any direction) from the average latency. We show that the expected time to reach such a state is polynomial in the inverse of the approximation parameters $\delta$ and $\epsilon$ as well as in the maximum elasticity of the latency functions, and logarithmic in the ratio between maximum and minimum potential. Hence, if the maximum latency of a path is fixed, the time is only logarithmic in the number of players and independent of the size of the strategy space and the number of resources.

We complement these results by various lower bounds. First, it is clear that pseudopolynomial time is required to reach exact imitation-stable states. This follows from the fact that there exist states in which all latency improvements are arbitrarily small, resulting in arbitrarily small migration probabilities. Hence, already a single step may take pseudopolynomially long. As a concept of approximate stable states one could have required all agents to be approximately satisfied, rather than only all but a $\delta$-fraction. This, however, would require to wait a polynomial number of rounds for the last agent to become approximately satisfied, as opposed to our logarithmic bound. Finally, we consider sequential imitation processes in which only one agent may move at a time. We extend a construction from [1] to show that there exist instances in which the shortest sequence of imitations that leads to an imitation-stable state is exponentially long.

The IMITATION PROTOCOL has one drawback: It is not innovative in the following sense. It might happen with small but non-zero probability that all players currently using the same strategy $P$ migrate towards other strategies and no other player migrates towards $P$. In this case, the knowledge about the existence of strategy $P$ is lost and cannot be regained. For singleton games, i. e., games in which each strategy is a singleton set, in which empty links have latency zero, we show in Section 5 that the probability of this event occurring in 
a polynomial number of rounds is negligible. This also has an important consequence: The cost of a state to which the IMITATION PROTOCOL converges is, on expectation, not much worse than the cost of a Nash equilibrium. More precisely, we show for the case of linear latency functions that the expected cost of a state to which the IMITATION PROTOCOL converges is within a constant factor of the optimal solution.

We conclude with a discussion of a possible extension of the IMITATION PROTOCOL in Section 6. In cases, in which convergence to a Nash equilibrium is required, it is possible to adjust the dynamics and occasionally let players use an EXPLORATION PROTOCOL. Using such a protocol, players sample other strategies directly instead of sampling them by looking at other players. We show that a suitable definition of such a protocol and a suitable combination with the IMITATION PROTOCOL guarantee convergence to Nash equilibria in the long run.

To the best of our knowledge, this is the first work that considers concurrent protocols for atomic congestion games that are not restricted to parallel links and linear latency functions.

\subsection{Related Work}

Rosenthal [24] proves that every congestion game possesses a Nash equilibrium, and that better response dynamics converge to Nash equilibria. In these dynamics players have complete knowledge, and, in every round, only a single player deviates to a better strategy than it currently uses. Fabrikant et al. [11], however, observe that, in general, from an appropriately chosen initial state it takes exponentially many steps until players finally reach an equilibrium. This negative result still holds in games with $\epsilon$-greedy players, i. e., in games in which players only deviate if their latency decreases by a relative factor of at least $1+\epsilon[1,7,26]$. Moreover, Fabrikant et al. [11] prove that, in general, computing a Nash equilibrium is PLS-complete. Their result still holds in the case of asymmetric network congestion games. In addition, Skopalik and Vöcking [26] prove that even computing an approximate Nash equilibrium is PLS-complete. On the positive side, best response dynamics converge quickly in singleton and matroid congestion games [1,21]. Additionally, Chien and Sinclair [7] consider the convergence time of best response dynamics to approximate Nash equilibria in symmetric games. They prove fast convergence to approximate Nash equilibria provided that the latency of a resource increases by at most a factor for each additional user. Finally, Goldberg [18] considers a protocol applied to a scenario where $n$ weighted users assign load to $m$ parallel links and the latency equals the load of a resource. In this protocol, randomly selected players move sequentially, and migrate to a randomly selected resource if this improves their latency. The expected time to reach a Nash equilibrium is pseudopolynomial. Results considering other protocols and links with latency functions are presented in [9]

The social cost of (approximate) Nash equilibria in congestion games has been subject to numerous studies. The most prominent concept has been the price of anarchy [22], which is the ratio of the worst cost of any Nash equilibrium over the cost of an optimal assignment. Roughgarden and Tardos [25] conducted the first study of general, non-atomic congestion games and showed a tight bound of $4 / 3$ for the price of anarchy with linear latency functions. For atomic games and linear latencies, Awerbuch et al. [2] and Christodoulou and Koutsoupias [8] show a tight bound of 2.5. The special case of (weighted) singleton games has been of particularly strong interest, and we refer the reader to [23, chapter 20] for an introduction to the numerous results. In terms of dynamics, Awerbuch et al. [3] consider the number of best-response steps required to reach a desirable state, which has a social cost only a constant factor larger than that of a social optimum. They show that even in congestion games with linear latencies there are exponentially long best-response sequences for reaching such a desirable state. In contrast, Fanelli et al. [12] show that for linear latency functions there are also much faster best response sequences that reach a desirable state after at most $\Theta(n \log \log n)$ steps.

Recently, concurrent protocols have been studied in various models and under various assumptions. EvenDar and Mansour [10] consider concurrent protocols in a setting where the links have speeds. However, their protocols require global knowledge in the sense that the users must be able to determine the set of underloaded and overloaded links. Given this knowledge, the convergence time is doubly logarithmic in the number of players. In [4] the authors consider a distributed protocol for the case that the latency equals the load that does not rely on this knowledge. Their bounds on the convergence time are also doubly logarithmic in the number of players but polynomial in the number of links. In [5] the results are generalized to the case of weighted jobs. In this case, the convergence time is only pseudopolynomial, i. e., polynomial in the number of users, 
links, and in the maximum weight. Finally, Fotakis et al. [17] consider a scenario with latency functions for every resource. Their protocol involves local coordination among the players sharing a resource. For the family of games in which the number of players asymptotically equals the number of resources they prove fast convergence to almost Nash equilibria. Intuitively, an almost Nash equilibrium is a state in which there are not too many too expensive and too cheap resources. In [13], a load balancing scenario is considered in which no information about the target resource is available. The authors present an efficient protocol in which the migration probability depends purely on the cost of the currently selected strategy.

In [15] the authors consider congestion games in the Wardrop model, where an infinite population of players carries an infinitesimal amount of load each. They consider a protocol similar to ours and prove that with respect to approximate equilibria it behaves like an FPTAS, i. e., it reaches an approximate equilibrium in time polynomial in the approximation parameters and the representation length of the instance (e.g., if the latency functions are polynomials in coefficient representation). In contrast to our work the analysis of the continuous model does not have to take into account probabilistic effects.

Our protocol is based on the notion of imitation, a concept frequently applied in evolutionary game theory. For an introduction to imitation dynamics, see, e g., [20, 27].

\section{Congestion Games and Imitation Dynamics}

In this section, we provide a formal description of our model. We define congestion games in terms of networks, that is, the strategy space of each player corresponds to the set of paths connecting a particular source-sink pair in a network. However, our results are independent of this definition and still hold in general, symmetric congestion games. Furthermore, we introduce the slope and the elasticity of latency functions, and give a precise definition of the IMITATION PROTOCOL.

\subsection{Symmetric Network Congestion Games}

A symmetric network congestion game is a tuple $\left(G,(s, t), \mathcal{N},\left(\ell_{e}\right)_{e \in E}\right)$, where $G=(V, E)$ denotes a network with vertices $V$ and $m$ directed edges $E$, and $s \in V$ and $t \in V$ denote a source and a sink vertex. Furthermore, $\mathcal{N}$ denotes a set of $n$ agents or players, and $\left(\ell_{e}\right)_{e \in E}$ a family of non-decreasing and differentiable latency functions $\ell_{e}: \mathbb{R}_{\geq 0} \rightarrow \mathbb{R}_{\geq 0}$. We assume that for all $e \in E$, the latency functions satisfy $\ell_{e}(x)>0$ for all $x>0$. The strategy space of all players equals the set of paths $\mathcal{P}$ connecting the source $s$ with the sink $t$. If $G$ consists of two nodes $s$ and $t$ only, which are connected by a set of parallel links, then we call the game a singleton game. A state $x$ of the game is a vector $\left(x_{P}\right)_{P \in \mathcal{P}}$ where $x_{P}$ denotes the number of players utilizing path $P$ in state $x$, and $x_{e}=\sum_{P \ni e} x_{P}$ is the congestion of edge $e \in E$ in state $x$. The latency of edge $e$ in state $x$ is given by $\ell_{e}\left(x_{e}\right)$, and the latency of path $P \in \mathcal{P}$ is

$$
\ell_{P}(x)=\sum_{e \in P} \ell_{e}\left(x_{e}\right) .
$$

The latency of a player is the latency of the path it chooses.

For brevity, for all $P \in \mathcal{P}$, let $1_{P}$ denote the $m$-dimensional unit vector with the one in position $P$. In state $x$ a player has an incentive to switch from path $P$ to path $Q$ if this would strictly decrease its latency, i. e., if

$$
\ell_{P}(x)>\ell_{Q}\left(x+1_{Q}-1_{P}\right) .
$$

If no player has an incentive to change its strategy, then $x$ is at a Nash equilibrium. It is well known [24], that the set of Nash equilibria corresponds to the set of states that minimize the potential function

$$
\Phi(x)=\sum_{e \in E} \sum_{i=1}^{x_{e}} \ell_{e}(i) .
$$


In the following, let $\Phi^{*}=\min _{x} \Phi(x)$ be the minimum potential. Note that due to our definition of the latency functions $\Phi^{*}>0$. For every path $P \in \mathcal{P}$ let

$$
\ell_{P}^{+}(x)=\ell_{P}\left(x+1_{P}\right) .
$$

Note that for every path $Q \in \mathcal{P}$

$$
\ell_{P}^{+}(x) \geq \ell_{P}\left(x+1_{P}-1_{Q}\right)
$$

Additionally, let

$$
L_{\mathrm{av}}(x)=\sum_{P \in \mathcal{P}} \frac{x_{P}}{n} \ell_{P}(x)
$$

denote the average latency of the paths in state $x$, and let

$$
L_{\mathrm{av}}^{+}(x)=\sum_{P \in \mathcal{P}} \frac{x_{P}}{n} \ell_{P}\left(x+1_{P}\right) .
$$

Finally, let $\ell_{\max }=\max _{x} \max _{P \in \mathcal{P}} \ell_{P}(x)$ denote the maximum latency of any path. Throughout this paper, whenever we consider a fixed state $x$ we simply drop the $\operatorname{argument}(x)$ from $\Phi, \ell_{P}, \ell_{P}^{+}, L_{\mathrm{av}}$, and $L_{\mathrm{av}}^{+}$.

\subsection{The Elasticity and the Slope of Latency Functions}

To bound the steepness of the latency functions and the effect that overshooting may have, we consider the elasticity of the latency functions. Let $d$ denote an upper bound on the elasticity of the latency functions, i. e.,

$$
d \geq \max _{e \in E} \sup _{x \in(0, n]}\left\{\frac{\ell_{e}^{\prime}(x) \cdot x}{\ell_{e}(x)}\right\} .
$$

Now given a latency function with elasticity $d$, it holds that for any $x$ and $\alpha \geq 1, \ell_{e}(\alpha x) \leq \ell_{e}(x) \cdot \alpha^{d}$ and for $0 \leq \alpha<1, \ell_{e}(\alpha x) \geq \ell_{e}(x) \cdot \alpha^{d}$. As an example, the function $a x^{d}$ has elasticity $d$.

For almost empty resources, we will also need an upper bound on the slope of the the latency functions. Let $\nu_{e}$ denote the maximum slope on almost empty edges, i.e.,

$$
\nu_{e}=\max _{x \in\{1, \ldots, d\}}\left\{\ell_{e}(x)-\ell_{e}(x-1)\right\} .
$$

Finally, for $P \in \mathcal{P}$, let $\nu_{P}=\sum_{e \in P} \nu_{e}$ and choose $\nu$ such that $\nu \geq \max _{P \in \mathcal{P}} \nu_{P}$.

\subsection{The Imitation Protocol}

Our Imitation Protocol (Protocol 1) proceeds in two steps. First, a player samples another agent uniformly at random. The player then migrates with a certain probability from its old path $P$ to the sampled path $Q$ depending on the anticipated relative latency gain $\left(\ell_{P}(x)-\ell_{Q}\left(x+1_{Q}-1_{P}\right)\right) / \ell_{P}(x)$ and on the elasticity of the latency functions. Our analysis concentrates on dynamics that result from the protocol being executed by the players in parallel in a round-based fashion. These dynamics generate a sequence of states $x(0), x(1), \ldots$. The resulting dynamics converge to a state that is stable in the sense that imitation cannot produce further progress, i. e., $x(t+1)=x(t)$ with probability 1 . Such a state is called an imitation-stable state. In other words, a state is imitation-stable if it is $\epsilon$-Nash with $\epsilon=\nu$ with respect to the strategy space restricted to the current support. Here, $\epsilon$-Nash means that no agent can improve its own payoff unilaterally by more than $\epsilon$.

As discussed in the introduction, the main difficulty in the design of the protocol is to bound overshooting effects. To get an intuition of this problem, consider two parallel links of which the first has the constant latency function $\ell_{1}(x)=c$ and the second has the latency function $\ell_{2}(x)=x^{d}$. Recall that the elasticity of $\ell_{2}$ is $d$. Furthermore, assume that only a small number of agents $x_{2}$ utilizes link 2 whereas the majority of $n-x_{2}$ users utilizes link 1 . Let $b=c-x_{2}^{d}>0$ denote the latency difference between the two links. 


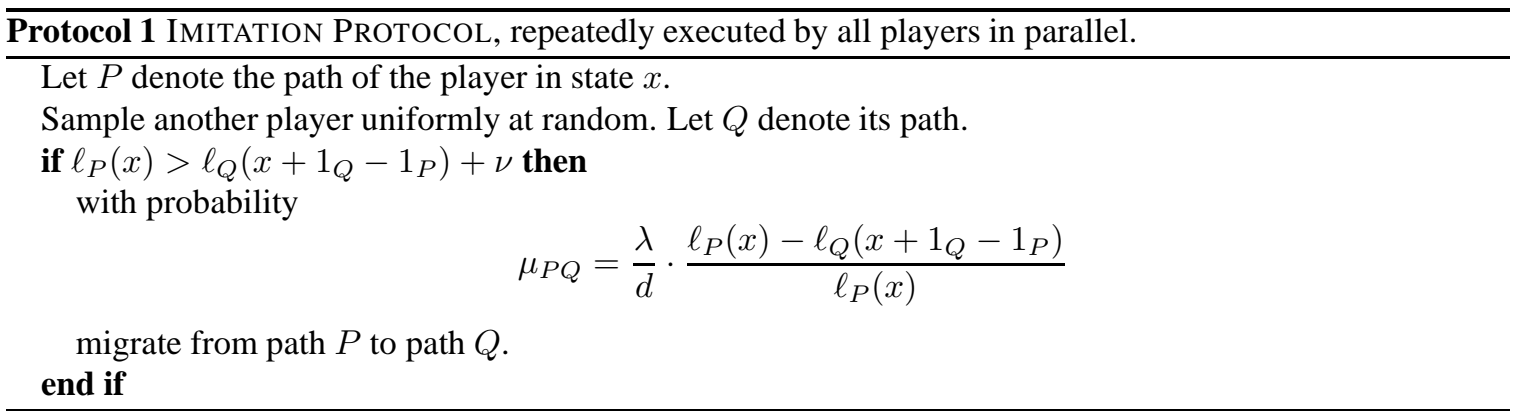

A simple calculation shows that using the protocol without the damping factor $1 / d$, the expected latency increase on link 2 would be $\Theta(b \cdot d)$, overshooting the balanced state by a factor $d$. For this reason, we reduce the migration probability accordingly. The constant $\lambda$ will be determined later.

Note that the arguments in the last paragraph hold for the expected load changes. Our protocol, however, has to take care of probabilistic effects, i. e., the realized migration vector may differ from its expectation. Typically, we can use the elasticity to bound the impact of this effect. However, if the congestion on an edge is very small, i. e., less than $d$, then the number of joining agents is not concentrated sharply enough around its expectation. In order to compensate for this, we add an additional requirement that agents only migrate if the anticipated latency gain is at least $\nu$ and use this to bound probabilistic effects if the congestion of the edge is less than $d$. Let us remark that we will see below (Theorem 9) that for a large class of singleton games it is very unlikely, that an edge will ever have a load of $d$ or less, so the protocol will behave in the same way with high probability for a polynomial number of rounds even if this additional requirement is dropped.

\section{Imitation Dynamics in Games with General Strategy Spaces}

In this chapter, we consider imitation dynamics that emerge if in each round players concurrently apply the IMITATION PROTOCOL. At first, we observe that imitation dynamics converge to imitation stable states since in each round the potential $\Phi(x)$ decreases in expectation. From this result we derive a pseudopolynomial upper bound on the convergence time to imitation-stable states.

\subsection{Pseudopolynomial Time Convergence to Imitation-Stable States}

Consider two states $x$ and $x^{\prime}$ as well as a migration vector $\Delta x=\left(\Delta x_{P}\right)_{P \in \mathcal{P}}$ such that $x^{\prime}=x+\Delta x$. We may imagine $\Delta x$ as the result of one round of the IMITATION PROTOCOL although the following lemma is independent of how $\Delta x$ is constructed. Furthermore, we consider $\Delta x$ to be composed of a set of migrations of agents between pairs of paths, i. e., $\Delta x_{P Q}$ denotes the number of players who switch from path $P$ to path $Q$, and $\Delta x_{P}$ denotes the total increase or decrease of the number of players utilizing path $P$, that is,

$$
\Delta x_{P}=\sum_{Q \in \mathcal{P}}\left(x_{Q P}-x_{P Q}\right)
$$

Also, let $\Delta x_{e}=\sum_{P \ni e} \Delta x_{P}$ denote the induced change of the number of players utilizing edge $e \in E$. In order to prove convergence, we define the virtual potential gain

$$
V_{P Q}(x, \Delta x)=x_{P Q} \cdot\left(\ell_{Q}\left(x+1_{Q}-1_{P}\right)-\ell_{P}(x)\right)
$$

which is the sum of the potential gains each player migrating from path $P$ to path $Q$ would contribute to $\Delta \Phi$ if each of them was the only migrating player. Note that if a player improves the latency of his path, the potential gain is negative. The sum of all virtual potential gains is a very rough lower bound on the true potential gain $\Delta \Phi(x, \Delta x)=\Phi(x+\Delta x)-\Phi(x)$. In order to compensate for the fact that players concurrently 
change their strategies, consider the error term on an edge $e \in E$ :

$$
F_{e}(x, \Delta x)= \begin{cases}\sum_{u=x_{e}+1}^{x_{e}+\Delta x_{e}} \ell_{e}(u)-\ell_{e}\left(x_{e}+1\right) & \text { if } \Delta x_{e}>0 \\ \sum_{u=x_{e}+\Delta x_{e}+1}^{x_{e}} \ell_{e}\left(x_{e}\right)-\ell_{e}(u) & \text { if } \Delta x_{e}<0 \\ 0 & \text { if } \Delta x_{e}=0\end{cases}
$$

Subsequently, we show that the sum of the virtual potential gains and the error terms is indeed an upper bound on the true potential gain $\Delta \Phi(x, \Delta x)$. A similar result is shown in [16] for a continuous model.

Lemma 1. For any assignment $x$ and migration vector $\Delta x$ it holds that

$$
\Delta \Phi(x, \Delta x) \leq \sum_{P, Q \in \mathcal{P}} V_{P Q}(x, \Delta x)+\sum_{e \in E} F_{e}(x, \Delta x) .
$$

Proof. We first express the virtual potential gain in terms of latencies on the edges. Clearly,

$$
\begin{aligned}
\sum_{P, Q \in \mathcal{P}} V_{P Q}(x, \Delta x) & =\sum_{P, Q \in \mathcal{P}} x_{P Q} \cdot\left(\ell_{Q}\left(x+1_{Q}-1_{P}\right)-\ell_{P}(x)\right) \\
& \leq \sum_{P, Q \in \mathcal{P}} x_{P Q} \cdot\left(\sum_{e \in Q} \ell_{e}\left(x_{e}+1\right)-\sum_{e \in P} \ell_{e}\left(x_{e}\right)\right) \\
& \leq \sum_{e: \Delta x_{e}>0} \Delta x_{e} \cdot \ell_{e}\left(x_{e}+1\right)+\sum_{e: \Delta x_{e}<0} \Delta x_{e} \cdot \ell_{e}\left(x_{e}\right) .
\end{aligned}
$$

The true potential gain, however, is

$$
\begin{aligned}
\Delta \Phi(x, \Delta x)= & \sum_{e: \Delta x_{e}>0} \sum_{u=x_{e}+1}^{x_{e}+\Delta x_{e}} \ell_{e}(u)-\sum_{e: \Delta x_{e}<0} \sum_{u=x_{e}-\Delta x_{e}+1}^{x_{e}} \ell_{e}(u) \\
= & \sum_{e: \Delta x_{e}>0}\left(\Delta x_{e} \cdot \ell_{e}\left(x_{e}+1\right)+\sum_{u=x_{e}+1}^{x_{e}+\Delta x_{e}}\left(\ell_{e}(u)-\ell_{e}\left(x_{e}+1\right)\right)\right) \\
& +\sum_{e: \Delta x_{e}<0}\left(\Delta x_{e} \cdot \ell_{e}\left(x_{e}\right)+\sum_{u=x_{e}-\Delta x_{e}+1}^{x_{e}}\left(\ell_{e}\left(x_{e}\right)-\ell_{e}(u)\right)\right) .
\end{aligned}
$$

Substituting Equation (1) for the left term of each sum and the definition of $F_{e}$ for the right term of each sum, we obtain the claim of the Lemma.

In the following, we consider $\Delta x$ to be a migration vector generated by the IMITATION PROTOCOL rather than an arbitrary vector. In this case, $\Delta x$ is a random variable and all probabilities and expectations are taken with respect to the IMITATION PROTOCOL. In order to prove that the potential decreases in expectation, we derive a bound on the size of the error terms. We show that the error terms reduce the virtual potential gain by at most a factor of two, or, put another way, that the true potential gain is at least half of the virtual potential gain.

Lemma 2. Let $x$ denote a state and let the random variable $\Delta x$ denote a migration vector generated by the IMITATION PROTOCOL. Then,

$$
\mathbb{E}[\Delta \Phi(x, \Delta x)] \leq \frac{1}{2} \sum_{P, Q \in \mathcal{P}} \mathbb{E}\left[V_{P Q}(x, \Delta x)\right] .
$$


Proof. For any given round, each term in $V_{P Q}, P, Q \in \mathcal{P}$ and $F_{e}, e \in E$ can be associated with an agent. Fix an agent $i$ migrating from, say, $P$ to $Q$. Its contribution to the $V_{P Q}(x, \Delta x)$ is $\ell_{Q}\left(x+1_{Q}-1_{P}\right)-\ell_{P}(x)$ (this is the same for all agents moving from $P$ to $Q$ ). It also contributes to $F_{e}, e \in P \cup Q$. The size of this term depends on the ordering of the agents. We will consider the migrating agents in ascending order of the migration probability $\mu_{P_{j} Q_{j}}$, where $P_{j}$ and $Q_{j}$ denote the origin and destination path of agent $j$, respectively. Ties are broken arbitrarily.

Fix an edge $e \in E$ and let $A^{+}(e)$ and $A^{-}(e)$ denote the set of agents migrating to and away from $e \in E$, respectively. Let $A(e)=A^{+}(e) \cup A^{-}(e)$. Let $\Delta \tilde{x}_{e}$ denote the contribution to $\Delta x_{e}$ of agents in $A(e)$ which occur in our ordering with respect to $\mu_{P Q}$ before agent $i$.

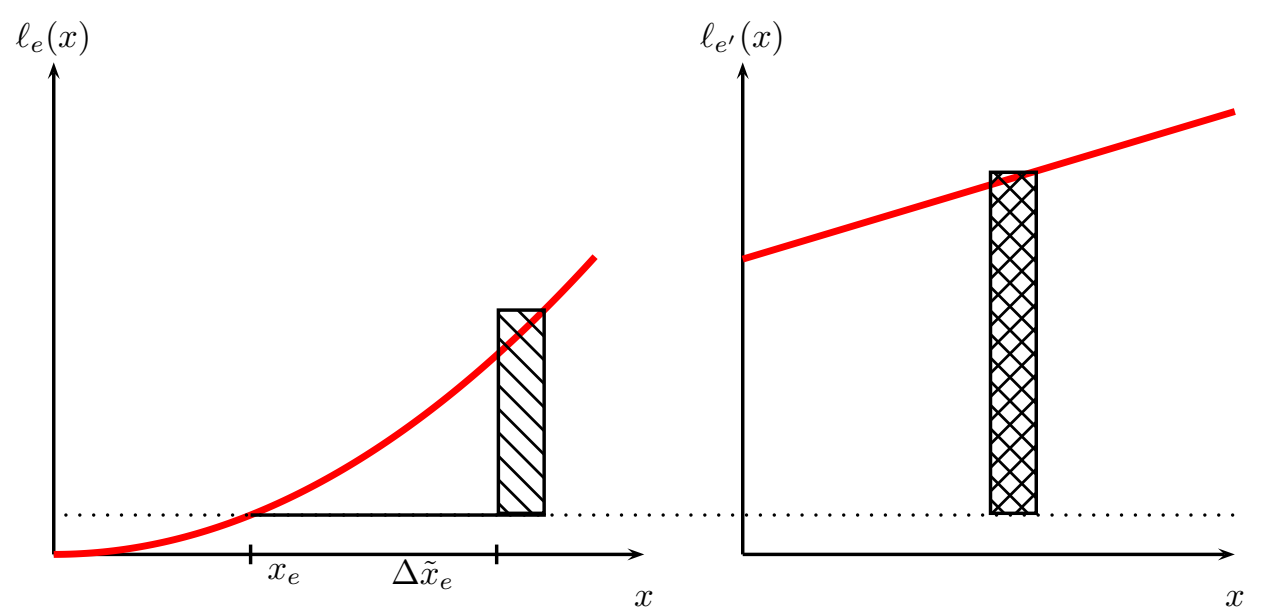

Figure 1: Potential gain of an agent migrating from edge $e^{\prime}$ towards edge $e$. The hatched area is the agent's virtual potential gain. The shaded area on the left is this agents contribution to the error term, caused by the $\Delta \tilde{x}_{e}$ agents ranking before the agent under consideration (with respect to $\mu_{P Q}$ ).

Agent $i$ 's contribution to $F_{e}(x, \Delta x)$ is $\Delta \tilde{\ell}_{e}\left(\Delta \tilde{x}_{e}\right)$ where we define the error function $\Delta \tilde{\ell}_{e}(\delta)=\ell_{e}\left(x_{e}+\right.$ $1+\delta)-\ell_{e}\left(x_{e}+1\right)$. For an illustration, see Figure 1. Note that there is an exception: If $e \in Q \cap P$, then the contribution of agent $i$ to $F_{e}$ is zero and there is nothing to show. For brevity, let us write $\ell_{e}=\ell_{e}\left(x_{e}\right)$ and $\ell_{e}^{+}=\ell_{e}\left(x_{e}+1\right)$ as well as $\ell_{P}=\ell_{P}(x)$ and $\ell_{Q}^{+}=\ell_{P}\left(x_{e}+1_{Q}-1_{P}\right)$. For $e \in Q \backslash P$ we show that

$$
\mathbb{E}\left[\Delta \tilde{\ell}_{e}\left(\Delta \tilde{x}_{e}\right)\right] \leq \frac{1}{8} \cdot\left(\ell_{P}-\ell_{Q}^{+}\right) \cdot\left(\frac{\ell_{e}^{+}}{\ell_{Q}^{+}}+\frac{\nu_{e}}{\nu_{Q}}\right),
$$

and for $e \in P \backslash Q$,

$$
\mathbb{E}\left[\Delta \tilde{\ell}_{e}\left(\Delta \tilde{x}_{e}\right)\right] \leq \frac{1}{8} \cdot\left(\ell_{P}-\ell_{Q}^{+}\right) \cdot\left(\frac{\ell_{e}}{\ell_{P}}+\frac{\nu_{e}}{\nu_{P}}\right) .
$$

Thus, the expected sum of the error terms of an agent migrating from $P$ to $Q$ is at most

$$
\frac{\ell_{P}-\ell_{Q}^{+}}{8}\left(\sum_{e \in P \backslash Q}\left(\frac{\ell_{e}}{\ell_{P}}+\frac{\nu_{e}}{\nu_{P}}\right)+\left(\sum_{e \in Q \backslash P} \frac{\ell_{e}^{+}}{\ell_{Q}^{+}}+\frac{\nu_{e}}{\nu_{Q}}\right)\right) \leq \frac{1}{2}\left(\ell_{P}-\ell_{Q}^{+}\right),
$$

i. e., half of its virtual potential gain, which proves the lemma. First, consider the case that $e \in Q$ where $Q$ denotes the destination path of agent $i$. 
For brevity, let us write $I_{P Q}=\left(\ell_{P}-\ell_{Q}^{+}\right) / \ell_{P}$ for the incentive to migrate from $P$ to $Q$. Again, consider the case that $e \in Q$ where $Q$ denotes the destination path of agent $i$. Then, due to our ordering of the agents,

$$
\mathbb{E}\left[\Delta \tilde{x}_{e}\right] \leq n \cdot \frac{x_{e}}{n} \cdot \mu_{P Q} \leq \frac{\lambda \cdot x_{e} \cdot I_{P Q}}{d},
$$

implying

$$
x_{e} \geq \frac{\mathbb{E}\left[\Delta \tilde{x}_{e}\right] \cdot d}{\lambda \cdot I_{P Q}} .
$$

Furthermore, due to the elasticity of $\ell_{e}$, and using $(1+1 / x)^{x} \leq \exp (1)$, we obtain

$$
\begin{aligned}
\Delta \tilde{\ell}_{e}(\delta) & \leq \ell_{e}^{+} \cdot\left(\frac{x_{e}+1+\delta}{x_{e}+1}\right)^{d}-\ell_{e} \\
& \leq \ell_{e}^{+} \cdot\left(1+\frac{\delta}{x_{e}}\right)^{d}-\ell_{e}^{+} \\
& \leq \ell_{e}^{+} \cdot\left(\mathrm{e}^{\frac{d \delta}{x_{e}}}-1\right) .
\end{aligned}
$$

Subsequently, we consider two cases.

Case 1: $\mathbb{E}\left[\Delta \tilde{x}_{e}\right] \geq \frac{1}{64}$. Substituting Inequality (5) into Inequality (6), we obtain for every $\kappa \in \mathbb{R}-\geq 0 q$

$$
\Delta \tilde{\ell}_{e}\left(\kappa \mathbb{E}\left[\Delta \tilde{x}_{e}\right]\right) \leq \ell_{e}^{+} \cdot\left(\mathrm{e}^{\kappa \lambda I_{P Q}}-1\right) .
$$

Now, note that for every $k \in \mathbb{N}$ and $\kappa \in[k, k+1]$

$$
\begin{aligned}
\mathbb{P}\left[\Delta \tilde{x}_{e} \geq \kappa \mathbb{E}\left[\Delta \tilde{x}_{e}\right]\right] & \leq \mathbb{P}\left[\Delta \tilde{x}_{e} \geq k \mathbb{E}\left[\Delta \tilde{x}_{e}\right]\right] \text { and } \\
\Delta \tilde{\ell}_{e}\left(\kappa \mathbb{E}\left[\Delta \tilde{x}_{e}\right]\right) & \leq \Delta \tilde{\ell}_{e}\left((k+1) \mathbb{E}\left[\Delta \tilde{x}_{e}\right]\right)
\end{aligned}
$$

hold. Applying a Chernoff bound (Fact 16 in the appendix), we obtain an upper bound for the expectation of $\mathbb{E}\left[\Delta \tilde{\ell}_{e}\left(\Delta \tilde{x}_{e}\right)\right]$ as follows.

$$
\begin{aligned}
\mathbb{E}\left[\Delta \tilde{\ell}_{e}\left(\Delta \tilde{x}_{e}\right)\right] & \leq \sum_{k=1}^{\infty} \mathbb{P}\left[\Delta \tilde{x}_{e} \geq k \mathbb{E}\left[\Delta \tilde{x}_{e}\right]\right] \cdot \Delta \tilde{\ell}_{e}\left((k+1) \mathbb{E}\left[\Delta \tilde{x}_{e}\right]\right) \\
& \leq \Delta \tilde{\ell}_{e}^{+}\left(5 \mathbb{E}\left[\Delta \tilde{x}_{e}\right]\right)+\sum_{k=5}^{\infty} \mathbb{P}\left[\Delta \tilde{x}_{e} \geq k \mathbb{E}\left[\Delta \tilde{x}_{e}\right]\right] \cdot \Delta \tilde{\ell}_{e}\left((k+1) \mathbb{E}\left[\Delta \tilde{x}_{e}\right]\right) \\
& \leq \ell_{e}^{+} \cdot\left(\mathrm{e}^{5 \lambda I_{P Q}}-1\right)+\sum_{k=5}^{\infty} \mathrm{e}^{-\frac{1}{4} \mathbb{E}\left[\Delta \tilde{x}_{e}\right] k \ln k} \cdot \ell_{e}^{+} \cdot\left(\mathrm{e}^{(k+1) \lambda I_{P Q}}-1\right) \\
& \leq \ell_{e}^{+} \cdot\left(\mathrm{e}^{5 \lambda I_{P Q}}-1\right)+\sum_{k=5}^{\infty} \mathrm{e}^{-\frac{1}{4} \mathbb{E}\left[\Delta \tilde{x}_{e}\right] k} \cdot \ell_{e}^{+} \cdot\left(\mathrm{e}^{2 k \lambda I_{P Q}}-1\right) \\
& \leq \ell_{e}^{+} \cdot\left(\mathrm{e}^{5 \lambda I_{P Q}}-1\right)+\int_{4}^{\infty} \mathrm{e}^{-\frac{1}{4} \mathbb{E}\left[\Delta \tilde{x}_{e}\right] u} \cdot \ell_{e}^{+} \cdot\left(\mathrm{e}^{2 u \lambda I_{P Q}}-1\right) d u \\
& =\ell_{e}^{+} \cdot\left(\mathrm{e}^{5 \lambda I_{P Q}}-1+\mathrm{e}^{-\mathbb{E}\left[\Delta \tilde{x}_{e}\right]} \frac{\mathrm{e}^{8 \lambda I_{P Q}}-1+\frac{8 \lambda I_{P Q}}{\mathbb{E}\left[\Delta \tilde{x}_{e}\right]}}{\frac{1}{4} \mathbb{E}\left[\Delta \tilde{x}_{e}\right]-2 \lambda I_{P Q}}\right)
\end{aligned}
$$


Now, due to Fact 17 in the appendix (with $r=1$ ) and our assumption that $\mathbb{E}\left[\Delta \tilde{x}_{e}\right] \geq 1 / 64$, we obtain

$$
\begin{aligned}
\mathbb{E}\left[\Delta \tilde{\ell}_{e}\left(\Delta \tilde{x}_{e}\right)\right] & \leq \lambda \cdot \ell_{e}^{+} \cdot I_{P Q} \cdot\left(5(\mathrm{e}-1)+\frac{8(\mathrm{e}-1)+8 \cdot 64}{\frac{1}{4 \cdot 64}-2 \lambda}\right) \\
& \leq c \cdot \lambda \cdot \ell_{e}^{+} \cdot \frac{\ell_{P}-\ell_{Q}^{+}}{\ell_{P}} \\
& \leq c \cdot \lambda \cdot \ell_{e}^{+} \cdot \frac{\ell_{P}-\ell_{Q}^{+}}{\ell_{Q}^{+}}
\end{aligned}
$$

for some constant $c$. The first inequality holds if $\lambda<1 / 512$, proving Equation (2) if $\lambda$ is chosen small enough.

Case 2: $\mathbb{E}\left[\Delta \tilde{x}_{e}\right]<\frac{1}{64}$. Again, in this case we can apply a Chernoff bound (Fact 16) to upper bound $\mathbb{E}\left[\Delta \tilde{\ell}_{e}\left(\Delta \tilde{x}_{e}\right)\right]$.

$$
\begin{aligned}
\mathbb{E}\left[\Delta \tilde{\ell}_{e}\left(\Delta \tilde{x}_{e}\right)\right] & \leq \sum_{k=1}^{n} \mathbb{P}\left[\Delta \tilde{x}_{e}=k\right] \cdot \Delta \tilde{\ell}_{e}(k) \\
& \leq \sum_{k=1}^{n} \mathbb{P}\left[\Delta \tilde{x}_{e} \geq \frac{k}{\mathbb{E}\left[\Delta \tilde{x}_{e}\right]} \mathbb{E}\left[\Delta \tilde{x}_{e}\right]\right] \cdot \Delta \tilde{\ell}_{e}(k) \\
& \leq \sum_{k=1}^{n} \mathrm{e}^{-k\left(\ln \left(k / \mathbb{E}\left[\Delta \tilde{x}_{e}\right]\right)-1\right)} \cdot \Delta \tilde{\ell}_{e}(k)
\end{aligned}
$$

There are two sub-cases:

Case 2a: $x_{e}>d$. In order to bound the expected latency increase, we apply the elasticity bound on $\ell_{e}$ :

$$
\begin{aligned}
\mathbb{E}\left[\Delta \tilde{\ell}_{e}\left(\Delta \tilde{x}_{e}\right)\right] & \leq \sum_{k=1}^{n} \mathrm{e}^{-k\left(\ln \left(k / \mathbb{E}\left[\Delta \tilde{x}_{e}\right]\right)-1\right)} \cdot \ell_{e}^{+} \cdot\left(\mathrm{e}^{\frac{k d}{x_{e}}}-1\right) \\
& \leq \ell_{e}^{+} \cdot \sum_{k=1}^{n} \mathrm{e}^{-k\left(\ln (k)-\ln \left(\mathbb{E}\left[\Delta \tilde{x}_{e}\right]\right)-1\right)} \cdot\left(\mathrm{e}^{\frac{k d}{x_{e}}}-1\right) \\
& \leq \ell_{e}^{+} \cdot \sum_{k=1}^{n}\left(\mathbb{E}\left[\Delta \tilde{x}_{e}\right]\left(\mathrm{e}^{k} \mathbb{E}\left[\Delta \tilde{x}_{e}\right]^{k-1}\right)\right) \mathrm{e}^{-k(\ln k)} \cdot\left(\mathrm{e}^{\frac{k d}{x_{e}}}-1\right) \\
& \leq \ell_{e}^{+} \cdot \mathbb{E}\left[\Delta \tilde{x}_{e}\right] \cdot \sum_{k=1}^{n} \mathrm{e}^{-k(\ln k)} \cdot\left(\mathrm{e}^{\frac{k d}{x_{e}}}-1\right) .
\end{aligned}
$$

Now, splitting up the sum, we define

$$
\begin{aligned}
L_{1} & =\mathbb{E}\left[\Delta \tilde{x}_{e}\right] \sum_{k=1}^{\left\lfloor\frac{8 x_{e}}{d}\right\rfloor} \mathrm{e}^{-k(\ln k)} \cdot\left(\mathrm{e}^{\frac{k d}{x_{e}}}-1\right) \\
& \leq \mathbb{E}\left[\Delta \tilde{x}_{e}\right] \frac{\left(\mathrm{e}^{8}-1\right) d}{8 x_{e}} \sum_{k=1}^{\left\lfloor\frac{8 x_{e}}{d}\right\rfloor} \mathrm{e}^{-k(\ln k) \cdot k} \\
L_{1} & \leq \frac{\mathrm{e}^{8}}{4} \cdot \mathbb{E}\left[\Delta \tilde{x}_{e}\right] \frac{d}{x_{e}} \\
& \leq \frac{\mathrm{e}^{8}}{4} \cdot \ell_{e}^{+} \cdot \lambda I_{P Q},
\end{aligned}
$$


where the first inequality uses the observation that $\mathrm{e}^{\frac{k d}{x_{e}}} \leq \mathrm{e}^{8}$ since $k \leq\left\lfloor 8 x_{e} / d\right\rfloor$, and Fact 17 (with $r=8)$. Additionally, where the third inequality uses the observation that $\sum_{k=1}^{\infty} \mathrm{e}^{-k(\ln k)} \cdot k \leq 2$, and finally where the last inequality uses Inequality (4).

For the second part of the sum, let

$$
\begin{aligned}
L_{2} & =\mathbb{E}\left[\Delta \tilde{x}_{e}\right] \sum_{k=\left\lceil\frac{8 x_{e}}{d}\right\rceil}^{\infty} \mathrm{e}^{-k(\ln k)} \cdot\left(\mathrm{e}^{\frac{k d}{x_{e}}}-1\right) \\
& \leq \mathbb{E}\left[\Delta \tilde{x}_{e}\right] \sum_{k=\left\lceil\frac{8 x_{e}}{d}\right\rceil}^{\infty} \mathrm{e}^{-k(\ln k)+\frac{k d}{x_{e}}} \\
& =\mathbb{E}\left[\Delta \tilde{x}_{e}\right] \sum_{k=\left\lceil\frac{8 x_{e}}{d}\right\rceil}^{\infty} \mathrm{e}^{\left.-k(\ln k-1) \quad \quad \text { (since } x_{e}>d\right)} \\
& \left.\leq \mathbb{E}\left[\Delta \tilde{x}_{e}\right] \sum_{k=\left\lceil\frac{8 x_{e}}{d}\right\rceil}^{\infty} \mathrm{e}^{-\frac{1}{2} k \ln k} \quad \text { (since } k \geq\left\lceil\frac{8 x_{e}}{d}\right\rceil \geq 8\right) \\
& \leq \mathbb{E}\left[\Delta \tilde{x}_{e}\right] \sum_{k=\left\lceil\frac{8 x_{e}}{d}\right\rceil}^{\infty}\left(\frac{d}{8 x_{e}}\right)^{\frac{1}{2} k} .
\end{aligned}
$$

Due to Fact 18 and since $x_{e}>d$

$$
\begin{aligned}
L_{2} & =\mathbb{E}\left[\Delta \tilde{x}_{e}\right] \frac{\left(\frac{d}{8 x_{e}}\right)^{\frac{8}{2}}}{1-\sqrt{\frac{d}{8 x_{e}}}} \\
& \leq \mathbb{E}\left[\Delta \tilde{x}_{e}\right] \frac{d}{x_{e}} \\
& \leq \lambda I_{P Q} .
\end{aligned}
$$

Reassembling the sum, we obtain

$$
\begin{aligned}
\mathbb{E}\left[\Delta \tilde{\ell}_{e}\left(\Delta \tilde{x}_{e}\right)\right] & \leq \ell_{e}^{+} \cdot\left(L_{1}+L_{2}\right) \\
& \leq \ell_{e}^{+} \cdot\left(\frac{\mathrm{e}^{8}}{4}+1\right) \lambda I_{P Q} .
\end{aligned}
$$

Again, by the same arguments as at the end of Case 1 this proves Equation (2) if $\lambda$ is less than $1 /\left(2 \mathrm{e}^{8}+8\right)$.

Case 2b: $x_{e} \leq d$. In this case we separate the upper bound on $\mathbb{E}\left[\Delta \tilde{\ell}_{e}\left(\Delta \tilde{x}_{e}\right)\right]$ into the section up to $d$ and above $d$. For the first section we use the fact that each additional player on resource $e$ causes a latency increase of at most $\nu_{e}$ as long as the load is at most $d$. We define the contribution to the expected latency increase by the events that up to $d-x_{e}$ join resource $e$, i. e., afterwards the 
congestion is still at most $d$. In this case, we may use $\nu_{e}$ to bound the contribution of each agent:

$$
\begin{aligned}
L_{1} & \leq \sum_{k=1}^{d-x_{e}} \mathrm{e}^{-k\left(\ln \left(\frac{k}{\mathbb{E}\left[\Delta \tilde{x}_{e}\right]}\right)-1\right)} \cdot k \nu_{e} \\
& \leq \mathrm{e} \nu_{e} \mathbb{E}\left[\Delta \tilde{x}_{e}\right]+\nu_{e} \mathbb{E}\left[\Delta \tilde{x}_{e}\right]^{2} \sum_{k=2}^{d-x_{e}} \mathrm{e}^{-k(\ln (k)-1)} \cdot k \\
& \leq \mathrm{e} \nu_{e} \mathbb{E}\left[\Delta \tilde{x}_{e}\right] \cdot\left(1+\frac{8 \mathbb{E}\left[\Delta \tilde{x}_{e}\right]}{\mathrm{e}}\right) \\
& \leq 3 \nu_{e} \mathbb{E}\left[\Delta \tilde{x}_{e}\right]
\end{aligned}
$$

where the third inequality holds since $\sum_{k=2}^{d-x_{e}} \mathrm{e}^{-k(\ln (k)-1)} \cdot k \leq 8$, and where the last inequality holds since $\mathbb{E}\left[\Delta \tilde{x}_{e}\right]<1 / 64$.

For the contribution of the agents increasing the load on resource $e$ to above $d$ we use the elasticity constraint again. This time, we do not consider the latency increase with respect to $\ell_{e}^{+}\left(x_{e}\right)$ but with respect to $\ell_{e}(d)$ :

$$
L_{2}=\sum_{k=d-x_{e}+1}^{n} \mathrm{e}^{-k \cdot\left(\ln \left(\frac{k}{\mathbb{E}\left[\Delta \tilde{x}_{e}\right]}\right)-1\right)} \cdot \ell_{e}(d) \cdot\left(\mathrm{e}^{\frac{d\left(k-\left(d-x_{e}\right)\right)}{d}}-1\right)
$$

As in case $(2 \mathrm{a})$

$$
\begin{aligned}
L_{2} & \leq \ell_{e}(d) \cdot \mathbb{E}\left[\Delta \tilde{x}_{e}\right] \cdot \sum_{k=d-x_{e}+1}^{\infty} \mathrm{e}^{-k \ln k+k-\left(d-x_{e}\right)} \\
& =\ell_{e}(d) \cdot \mathbb{E}\left[\Delta \tilde{x}_{e}\right] \cdot \sum_{k=1}^{\infty} \mathrm{e}^{-\left(k+\left(d-x_{e}\right)\right) \ln \left(k+\left(d-x_{e}\right)\right)+k} \\
& =\ell_{e}(d) \cdot \mathbb{E}\left[\Delta \tilde{x}_{e}\right] \cdot \mathrm{e}^{-\left(d-x_{e}\right)} \cdot \sum_{k=1}^{\infty} \mathrm{e}^{-\left(k+\left(d-x_{e}\right)\right) \ln \left(k+\left(d-x_{e}\right)\right)+k+d-x_{e}}
\end{aligned}
$$

Consider the series in the above expression as a function of $u=\left(d-x_{e}\right)$ and denote it by $S(u)$. Note that $S(u)$ converges for every $u \geq 0$ and $S(u) \rightarrow 0$ as $u \rightarrow \infty$. In particular, $S(u)<8$ for any $u \geq 0$, so

$$
\begin{aligned}
L_{2} & \leq 8 \ell_{e}(d) \cdot \mathbb{E}\left[\Delta \tilde{x}_{e}\right] \cdot \mathrm{e}^{-\left(d-x_{e}\right)} \\
& \leq 8\left(\ell_{e}\left(x_{e}\right)+\left(d-x_{e}\right) \nu_{e}\right) \cdot \mathbb{E}\left[\Delta \tilde{x}_{e}\right] \cdot \mathrm{e}^{-\left(d-x_{e}\right)}
\end{aligned}
$$

Since $\left(d-x_{e}\right) \cdot \mathrm{e}^{-\left(d-x_{e}\right)}<1 / 2$,

$$
L_{2} \leq 4\left(\ell_{e}\left(x_{e}\right)+\nu_{e}\right) \cdot \mathbb{E}\left[\Delta \tilde{x}_{e}\right]
$$

Altogether,

$$
\begin{aligned}
\mathbb{E}\left[\Delta \tilde{\ell}_{e}\left(\Delta \tilde{x}_{e}\right)\right] & \leq L_{1}+L_{2} \\
& \leq 7 \nu_{e} \mathbb{E}\left[\Delta \tilde{x}_{e}\right]+4 \ell_{e}\left(x_{e}\right) \mathbb{E}\left[\Delta \tilde{x}_{e}\right] \\
& \leq 7 \nu_{e} \mathbb{E}\left[\Delta \tilde{x}_{e}\right]+4 \frac{\lambda x_{e} I_{P Q}}{d} \cdot \ell_{e}\left(x_{e}\right) \\
& \leq \frac{7}{64} \nu \frac{\nu_{e}}{\nu_{Q}}+\frac{4 \lambda x_{e} I_{P Q}}{d} \cdot \ell_{e}\left(x_{e}\right)
\end{aligned}
$$


where we have used Equation (4) for the third inequality, and the inequalities $\mathbb{E}\left[\Delta \tilde{x}_{e}\right]<1 / 64$ and $\nu \geq \nu_{Q}$ for the last step. Since $x_{e} \leq d$ and $\ell_{P}-\ell_{Q}^{+} \geq \nu$,

$$
\mathbb{E}\left[\Delta \tilde{\ell}_{e}\left(\Delta \tilde{x}_{e}\right)\right] \leq \frac{1}{8}\left(\ell_{P}-\ell_{Q}^{+}\right) \frac{\nu_{e}}{\nu_{Q}}+\frac{4 \lambda\left(\ell_{P}-\ell_{Q}^{+}\right)}{\ell_{P}} \cdot \ell_{e}\left(x_{e}\right)
$$

again proving Equation (2) if $\lambda \leq 1 / 32$.

Finally, the case $e \in P$ is very similar.

Note that all migrating players add a negative contribution to the virtual potential gain since they migrate only from paths with currently higher latency to paths with lower latency. Hence, together with Lemma 2, we can derive the next corollary.

Corollary 3. Consider a symmetric network congestion game $\Gamma$ and let $x$ and $x^{\prime}$ denote states of $\Gamma$ such that $x^{\prime}$ is a random state generated after one round of executing the IMITATION PROTOCOL. Then,

$$
\mathbb{E}\left[\Phi\left(x^{\prime}\right)\right] \leq \Phi(x)
$$

with strict inequality as long as $x$ is not imitation-stable. Thus, $\Phi$ is a super-martingale.

It is obvious that the sequence of states generated by the IMITATION PROTOCOL terminates at an imitationstable state. From Lemma 2 we can immediately derive an upper bound on the time to reach such a state. However, since for arbitrary latency functions the minimum possible latency gain may be very small, this bound can clearly be only pseudo-polynomial. To see this, consider a state in which only one player can make an improvement. Then, the expected time until the player moves is inverse proportional to its latency gain.

Theorem 4. Consider a symmetric network congestion game in which all players use the IMITATION PROTOCOL. Let $x$ denote the initial state of the dynamics. Then the dynamics converge to an imitation-stable state in expected time

$$
\mathcal{O}\left(\frac{d n \ell_{\max } \Phi(x)}{\nu^{2}}\right)
$$

Proof. By definition of the Imitation Protocol, the expected virtual potential gain in any state $x^{\prime}$ which is not yet imitation-stable is at least

$$
\mathbb{E}\left[\sum_{P, Q \in \mathcal{P}} V_{P Q}\left(x^{\prime}, \Delta x^{\prime}\right)\right] \leq-\nu \cdot \frac{\lambda}{d n} \cdot \frac{\nu}{\ell_{\max }} .
$$

Hence, also the expected potential gain $\mathbb{E}\left[\Delta \Phi\left(x^{\prime}\right)\right]$ in every intermediate state $x^{\prime}$ of the dynamics is bounded from above by at least half of the above value. From this, it follows, that the expected time until the potential drops from at most $\Phi(x)$ to the minimum potential $\Phi^{*}$ is at most

$$
\frac{d n \ell_{\max }\left(\Phi(x)-\Phi^{*}\right)}{\lambda \nu^{2}}
$$

Formally, this is a consequence of Lemma 20 which can be found in the Appendix.

It is obvious that this result cannot be significantly improved since we can easily construct an instance and a state such that the only possible improvement that can be made is $\nu$. Hence, already a single step takes pseudopolynomially long. In case of polynomial latency functions Theorem 4 reads as follows. 
Corollary 5. Consider a symmetric network congestion game with polynomial latency functions with maximum degree $d$ and minimum and maximum coefficients $a_{\min }$ and $a_{\max }$, respectively. Let $k=\max _{P \in \mathcal{P}}|P|$. Then the dynamics converges to an imitation-stable state in expected time

$$
\mathcal{O}\left(d^{2} k^{2} n^{2 d+2} \cdot\left(\frac{a_{\max }}{a_{\min }}\right)^{2}\right) .
$$

Let us remark that all proofs in this section do not rely on the assumption that the underlying congestion game is symmetric. In fact, the lemma also holds for asymmetric congestion games in which each player samples only among players that have the same strategy space.

\subsection{Sequential Imitation Dynamics and a Lower Bound}

In the previous section, we proved that players applying the IMITATION PROTOCOL reach an imitation-stable state after a pseudopolynomial number of rounds. Recall that in this case each player decreases its latency by at least $\nu$ if it were the only player to change its strategy. In this section, we consider sequential imitation dynamics such that in each round a single player is permitted to imitate someone else. Furthermore, we assume that each player changes its path regardless of the anticipated latency gain. Now, it is obvious that sequential imitation dynamics converge towards imitation-stable states as the potential $\Phi$ strictly decreases after every strategy change. Hence, we focus on the convergence time of such dynamics.

For such sequential imitation dynamics we prove an exponential lower bound on the number of rounds to reach an imitation-stable state. To be precise, we present a family of symmetric network congestion games with corresponding initial states such that every sequence of imitation leading to an imitation-stable state is exponentially long. To some extent, this results complements Theorem 4 as it presents an exponential lower bound in a slightly different model. However, in this lower bound $\nu$ is arbitrary large and almost every state is imitation-state with respect to the IMITATION PROTOCOL.

Theorem 6. For every $n \in \mathbb{N}$, there exists a symmetric network congestion game with $n$ players, initial state $S^{\text {init }}$, polynomial bounded network size, and linear latency functions such that every sequential imitation dynamics that start in $S^{\text {init }}$ is exponentially long.

Subsequently, we do not give a complete proof of the theorem but we discuss how to adapt a series of constructions as presented in [1] which shows that there exists a family of symmetric network congestion games with the same properties as stated in the above theorem such that every best response dynamics starting in $S^{\text {init }}$ is exponentially long. To be precise, they prove that in every intermediate state of the best response dynamics exactly one player can improve its latency. Recall that in best response dynamics players know the entire strategy space and that in each round one player is permitted the switch to the best available path.

In the following, we summarize the constructions presented in [1]. At first, a PLS-reduction from the local search variant of MaxCut to threshold games is presented. In a threshold game, each player either allocates a single resource on its own or shares a bunch of resources with other players. Hence, in a threshold game each player chooses between two strategies only. The precise definition of these games is given below. Then, a PLS-reduction from threshold games to asymmetric network congestion games is presented. Finally, the authors of [1] show how to transform an asymmetric network congestion game into a symmetric one such that the desired properties of best response dynamics are preserved. All PLS-reductions are embedding, and there exists a family of instances of MaxCut with corresponding initial configurations such that in every intermediate configuration generated by a local search algorithm exactly one node can be moved to the other side of the cut. Therefore, there exists a family of symmetric network congestion games with the properties as stated above.

A naive approach to prove a lower bound on the convergence time of imitation dynamics in symmetric network congestion games is as follows. Building upon the lower bound of the convergence time of best responses dynamics, a player for every path is added to the game. Then the latency functions are adopted accordingly. However, in this case we would introduce an exponential number of additional players. In threshold games, however, the players' strategy spaces have size two only. Hence, we could apply this 
approach to threshold games. In the following, we present the details of this approach. It is then not difficult to verify that the PLS-reductions mentioned above can be reworked in order to prove Theorem 6. However, note that this does not imply that computing a imitation-stable state is PLS-complete since one can always assign all players to the same strategy which obviously is an imitation-stable state.

Threshold games are a special class of congestion games in which the set of resources $\mathcal{R}$ can be divided into two disjoint sets $\mathcal{R}_{\text {in }}$ and $\mathcal{R}_{\text {out }}$. The set $\mathcal{R}_{\text {out }}$ contains exactly one resource $r_{i}$ for every player $i \in \mathcal{N}$. This resource has a fixed latency $T_{i}$ called the threshold of player $i$. Each player $i$ has only two strategies, namely a strategy $S_{i}^{\text {out }}=\left\{r_{i}\right\}$ with $r_{i} \in \mathcal{R}_{\text {out }}$, and a strategy $S_{i}^{\text {in }} \subseteq \mathcal{R}_{\text {in. }}$. The preferences of player $i$ can be described in a simple and intuitive way: Player $i$ prefers strategy $S_{i}^{\text {in }}$ to strategy $S_{i}^{\text {out }}$ if the latency of $S_{i}^{\text {in }}$ is smaller than the threshold $T_{i}$. Quadratic threshold games are a subclass of threshold games in which the set $\mathcal{R}_{\text {in }}$ contains exactly one resource $r_{i j}$ for every unordered pair of players $\{i, j\} \subseteq \mathcal{N}$. Additionally, for every player $i \in \mathcal{N}$ of a quadratic threshold game, $S_{i}^{\text {in }}=\left\{r_{i j} \mid j \in \mathcal{N}, j \neq i\right\}$. Moreover, for every resource $r_{i j} \in \mathcal{R}_{\text {in }}: \ell_{r_{i j}}(x)=a_{i, j} \cdot x$ with $a_{i j} \in \mathbb{N}$, and for every resource $r_{i}: \ell_{r_{i}}(x)=1 / 2 \sum_{j \neq i} a_{i j} \cdot x$ to $r_{i}$.

Let $\Gamma$ be a quadratic treshold game that has an initial state $S^{\text {init }}$, such that every best response dynamics which starts $S^{\text {init }}$ is exponentially long, and every intermediate state has a unique player which can improve its latency. Suppose now that we replace every player $i$ in $\Gamma$ by three players $i_{1}, i_{2}$ and $i_{3}$ which all have the same strategy spaces as player $i$ has. Additionally, suppose that we choose new latency functions $\ell^{\prime}$ for every resource $r_{i}$ as follows: $\ell_{r_{i}}^{\prime}(x)=1 / 2 \sum_{j \neq i} a_{i j} \cdot x+3 / 2 \sum_{j \neq i} a_{i j}$. Hence, we add an additional offset of $3 / 2 \sum_{j \neq i} a_{i j}$.

Suppose now that we assign every player $i_{1}$ to $S_{i}^{\text {out }}$, and every player $i_{2}$ to $S_{i}^{\text {in }}$. For every possible strategy that the $i_{3}$ players can use, their latency increases by $2 \sum_{j \neq i} a_{i j}$, compared to the equivalent state in the original game, in which every player $i$ chooses the same strategy as player $i_{3}$ does. Hence, if if we assign every player $i_{3}$ to the strategy chosen by player $i$ in $S^{\text {init }}$ and if the players $i_{1}$ and $i_{2}$ were not permitted to change their strategies, then we would obtain the desired lower bound on the convergence time of imitation dynamics in threshold games. However, since also $i_{1}$ and $i_{2}$ are permitted to imitate, it remains to show that whenever player $i_{3}$ has changed its strategy, then both $i_{1}$ and $i_{2}$ do not want to change their strategies anymore.

First, suppose that player $i_{3}$ switches from the strategy of player $i_{2}$ to the strategy of player $i_{1}$. Obviously, player $i_{1}$ does not want to change its strategy as otherwise $i_{3}$ would not have imitated $i_{1}$. Suppose now that $i_{2}$, whose strategy is dropped by $i_{3}$, also wants to imitate $i_{1}$. In this case all three players would allocate $S_{i}^{\text {out }}$, and hence have latency $3 \sum_{r \in j \neq i} a_{i j}$. However, if player $i_{2}$ would stay with strategy $S^{\text {in }}$ then its latency is upper bounded by $2 \sum_{r \in S_{i}^{\text {in }}} a_{i j}$. Hence, players $i_{1}, i_{2}, i_{3}$ will never select $S^{\text {out }}$ at the same time.

Second, suppose that player $i_{3}$ switches from the strategy of player $i_{1}$ to the strategy of player $i_{2}$. Now, player $i_{2}$ does not want to change its strategy as otherwise $i_{3}$ would not have imitated $i_{2}$. Suppose now that $i_{1}$, whose strategy is dropped by $i_{3}$, also wants to imitate $i_{3}$. In this case, the latency would increase to at least $3 \sum_{r \in j \neq i} a_{i j}$, whereas player $i_{1}$ would have latency $2 \sum_{r \in j \neq i} a_{i j}$ if it would stay with strategy $S^{\text {out }}$. Hence, players $i_{1}, i_{2}, i_{3}$ will never select $S^{\text {in }}$ at the same time.

By applying the argument that all three players never allocate the same strategy at the same point in time we can conclude our claim and Theorem 6 follows.

\section{Fast Convergence to Approximate Equilibria}

Theorem 4 guarantees convergence of concurrent imitation dynamics generated by the IMITATION PROTOCOL to an imitation-stable state in the long run. However, it does not give a reasonable bound on the time due to the small progress that can be made. Hence, as our main result, we present bounds on the time to reach an approximate equilibrium. Here we relax the definition of an imitation-stable state in two aspects: We allow only a small minority of agents to deviate by more than a small amount from the average latency. Our notion of an approximate equilibrium is similar to the notion used in $[6,15,17]$. It is motivated by the following observation. When sampling other players each player gets to know its latency if it would adopt that players' strategy. Hence to some extend each player can compute the average latency $L_{\mathrm{av}}^{+}$and determine if its own latency is above or below that average. 
Definition $1\left((\delta, \epsilon, \nu)\right.$-equilibrium). Given a state $x$, let the set of expensive paths be $\mathcal{P}_{\epsilon, \nu}^{+}=\{P \in \mathcal{P}$ : $\left.\ell_{P}(x)>(1+\epsilon) L_{a v}^{+}+\nu\right\}$ and let the set of cheap paths be $\mathcal{P}_{\epsilon, \nu}^{-}=\left\{P \in \mathcal{P}: \ell_{P}(x)<(1-\epsilon) L_{a v}-\nu\right\}$. Let $\mathcal{P}_{\epsilon, \nu}=\mathcal{P}_{\epsilon, \nu}^{+} \cup \mathcal{P}_{\epsilon, \nu}^{-}$. A configuration $x$ is at a $(\delta, \epsilon, \nu)$-equilibrium iff it holds that $\sum_{P \in \mathcal{P} \epsilon, \nu} x_{P} \leq \delta \cdot n$.

Intuitively, a state at $(\delta, \epsilon, \nu)$-equilibrium is a state in which almost all agents are almost satisfied when comparing their own situation with the situation of other agents. One may hope that it is possible to reach a state in which all agents are almost satisfied quickly. This would be a relaxation of the concept of Nash equilibrium. We will argue below, however, that there is no rapid convergence to such states.

Theorem 7. For an arbitrary initial assignment $x_{0}$, let $\tau$ denote the first round in which the IMITATION PROTOCOL reaches a $(\delta, \epsilon, \nu)$-equilibrium. Then,

$$
\mathbb{E}[\tau]=\mathcal{O}\left(\frac{d}{\epsilon^{2} \delta} \cdot \log \left(\frac{\Phi\left(x_{0}\right)}{\Phi^{*}}\right)\right)
$$

Proof. We consider a state $x(t)$ that is not at a $(\delta, \epsilon, \nu)$-equilibrium and derive a lower bound on the expected potential gain. There are two cases. Either at least half of the agents utilizing paths in $\mathcal{P}_{\epsilon, \nu}$ utilize paths in $\mathcal{P}_{\epsilon, \nu}^{+}$or at least half of them utilize paths in $\mathcal{P}_{\epsilon, \nu}^{-}$.

Case 1: Many agents use expensive paths, i.e., $\sum_{P \in \mathcal{P}_{\epsilon, \nu}^{+}} x_{P} \geq \delta n / 2$. Let us define the volume $T$ and the average ex-post latency $C$ of potential destination paths, i. e., paths with ex-post latency at most $(1+\epsilon) L_{\mathrm{av}}^{+}$, by

$$
T=\sum_{Q: \ell_{Q}^{+} \leq(1+\epsilon) L_{\mathrm{av}}^{+}} \frac{x_{Q}}{n} \quad \text { and } \quad C=\frac{1}{T} \sum_{Q: \ell_{Q}^{+} \leq(1+\epsilon) L_{\mathrm{av}}^{+}} \frac{x_{Q}}{n} \ell_{Q}^{+} .
$$

Clearly,

$$
L_{\mathrm{av}}^{+}=\sum_{P} \frac{x_{P}}{n} \ell_{P}^{+} \geq T \cdot C+(1-T) \cdot(1+\epsilon) L_{\mathrm{av}}^{+},
$$

and solving for $T$ yields

$$
T \geq \frac{\epsilon L_{\mathrm{av}}^{+}}{(1+\epsilon) L_{\mathrm{av}}^{+}-C}
$$

We now give a lower bound on the expected virtual potential gain given that the current state is not at a $(\delta, \epsilon, \nu)$-equilibrium. We consider only the contribution of agents utilizing paths in $\mathcal{P}_{\epsilon, \nu}^{+}$and sampling paths with ex-post latency below $(1+\epsilon) L_{\mathrm{av}}^{+}$. Then,

$$
\begin{aligned}
\mathbb{E}\left[\sum_{P, Q} V_{P Q}\right] & \leq-\frac{\lambda}{d} \sum_{P \in \mathcal{P}_{\epsilon, \nu}^{+}} x_{P} \sum_{Q: \ell^{+} \leq(1+\epsilon) L_{\mathrm{av}}^{+}} \frac{x_{Q}}{n} \cdot \frac{\ell_{P}-\ell_{Q}\left(x+1_{Q}-1_{P}\right)}{\ell_{P}}\left(\ell_{P}-\ell_{Q}\left(x+1_{Q}-1_{P}\right)\right) \\
& =-\frac{\lambda}{d} \sum_{P \in \mathcal{P}_{\epsilon, \nu}^{+}} x_{P} \ell_{P} \sum_{Q: \ell^{+} \leq(1+\epsilon) L_{\mathrm{av}}^{+}} \frac{x_{Q}}{n} \cdot\left(\frac{\ell_{P}-\ell_{Q}^{+}}{\ell_{P}}\right)^{2} .
\end{aligned}
$$

Using Jensen's inequality (Fact 19) and substituting $\ell_{P} \geq L_{\text {av }}^{+}$yields

$$
\mathbb{E}\left[\sum_{P, Q} V_{P Q}\right] \leq-\frac{\lambda}{d} L_{\mathrm{av}}^{+} \sum_{P \in \mathcal{P}_{\epsilon, \nu}^{+}} x_{P}\left(\sum_{Q: \ell^{+} \leq(1+\epsilon) L_{\mathrm{av}}^{+}} \frac{x_{Q}}{n} \cdot \frac{\ell_{P}-\ell_{Q}^{+}}{\ell_{P}}\right)^{2} \cdot \frac{1}{\sum_{Q: \ell_{Q}^{+} \leq(1+\epsilon) L_{\mathrm{av}}^{+}} \frac{x_{Q}}{n}} .
$$

Now we substitute $\ell_{P} \geq(1+\epsilon) L_{\mathrm{av}}^{+}$and use the fact that the squared expression is monotone in $\ell_{P}$. 
Furthermore, we substitute the definition of $T$ and $C$ to obtain

$$
\begin{aligned}
\mathbb{E}\left[\sum_{P, Q} V_{P Q}\right] & \leq-\frac{\lambda}{d} L_{\mathrm{av}}^{+} \sum_{P \in \mathcal{P}_{\epsilon, \nu}^{+}} x_{P}\left(\frac{T(1+\epsilon) L_{\mathrm{av}}^{+}-\sum_{Q: \ell^{+} \leq(1+\epsilon) L_{\mathrm{av}}^{+}} \frac{x_{Q} \ell_{Q}^{+}}{n}}{(1+\epsilon) L_{\mathrm{av}}^{+}}\right)^{2} \cdot \frac{1}{T} \\
& \leq-\frac{\lambda}{d} L_{\mathrm{av}}^{+} \sum_{P \in \mathcal{P}_{\epsilon, \nu}^{+}} x_{P}\left(\frac{T(1+\epsilon) L_{\mathrm{av}}^{+}-T C}{(1+\epsilon) L_{\mathrm{av}}^{+}}\right)^{2} \cdot \frac{1}{T} \\
& =-\frac{\lambda}{d} L_{\mathrm{av}}^{+} \cdot\left(\frac{(1+\epsilon) L_{\mathrm{av}}^{+}-C}{(1+\epsilon) L_{\mathrm{av}}^{+}}\right)^{2} \cdot T \cdot \sum_{P \in \mathcal{P}_{\epsilon, \nu}^{+}} x_{P} .
\end{aligned}
$$

We can now use the tradeoff shown in Equation (7), $C \leq L_{\mathrm{av}}^{+}$, and $\sum_{P \in \mathcal{P}_{\epsilon, \nu}^{+}} x_{P}>\delta n / 2$ to obtain

$$
\begin{aligned}
\mathbb{E}\left[\sum_{P, Q} V_{P Q}\right] & \leq-\frac{\lambda}{d} \cdot L_{\mathrm{av}}^{+} \cdot \frac{(1+\epsilon) L_{\mathrm{av}}^{+}-C}{\left((1+\epsilon) L_{\mathrm{av}}^{+}\right)^{2}} \cdot \epsilon L_{\mathrm{av}}^{+} \cdot \sum_{P \in \mathcal{P}_{\epsilon, \nu}^{+}} x_{P} \\
& \leq-\frac{\lambda}{d} \cdot \epsilon \cdot \frac{\epsilon L_{\mathrm{av}}^{+}}{(1+\epsilon)^{2}} \cdot \frac{\delta n}{2} \\
& \leq-\Omega\left(\frac{\epsilon^{2} \cdot \delta}{d} \cdot n L_{\mathrm{av}}^{+}\right) .
\end{aligned}
$$

Since $n L_{\mathrm{av}}^{+} \geq \Phi$, we have by Lemma 2

$$
\mathbb{E}[\Phi(x(t+1))] \leq \Phi(x(t))-\frac{1}{2} \mathbb{E}\left[\sum_{P, Q} V_{P Q}\right] \leq \Phi(x(t))\left(1-\Omega\left(\frac{\epsilon^{2} \cdot \delta}{d}\right)\right) .
$$

Case 2: Many agents use cheap paths, i.e., $\sum_{P \in \mathcal{P}_{\epsilon, \nu}^{-}} x_{P} \geq \delta n / 2$. This time, we define the volume $T$ and average latency $C$ of paths which are potential origins of agents migrating towards $\mathcal{P}_{\epsilon, \nu}^{-}$.

$$
T=\sum_{Q: \ell_{Q} \geq(1-\epsilon) L_{\mathrm{av}}} \frac{x_{Q}}{n} \text { and } C=\frac{1}{T} \sum_{Q: \ell_{Q} \geq(1-\epsilon) L_{\mathrm{av}}} \frac{x_{Q}}{n} \ell_{Q}
$$

This time,

$$
L_{\mathrm{av}} \leq T \cdot C+(1-T) \cdot(1-\epsilon) L_{\mathrm{av}}
$$

implying

$$
T \geq \frac{\epsilon L_{\mathrm{av}}}{C-(1-\epsilon) L_{\mathrm{av}}}
$$

Similarly as in Case 1 we now give a lower bound on the contribution to the virtual potential gain caused by agents with latency at least $(1-\epsilon) L_{\mathrm{av}}$ sampling agents in $\mathcal{P}_{\epsilon, \nu}^{-}$.

$$
\mathbb{E}\left[\sum_{P, Q} V_{P Q}\right] \leq-\frac{\lambda}{d} \sum_{Q: \ell_{Q} \geq(1-\epsilon) L_{\mathrm{av}}} x_{Q} \ell_{Q} \sum_{P \in \mathcal{P}_{\epsilon, \nu}^{-}} \frac{x_{P}}{n} \cdot\left(\frac{\ell_{Q}-\ell_{P}^{+}}{\ell_{Q}}\right)^{2} .
$$


we rearrange the sum, apply Jensen's inequality (Fact 19) to obtain

$$
\begin{aligned}
\mathbb{E}\left[\sum_{P, Q} V_{P Q}\right] & \leq-\frac{\lambda}{d} \sum_{P \in \mathcal{P}_{\epsilon, \nu}^{-}} x_{P} \sum_{Q: \ell_{Q} \geq(1-\epsilon) L_{\mathrm{av}}} \frac{x_{Q} \ell_{Q}}{n} \cdot\left(\frac{\ell_{Q}-\ell_{P}^{+}}{\ell_{Q}}\right)^{2} \\
& \leq-\frac{\lambda}{d} \sum_{P \in \mathcal{P}_{\epsilon, \nu}^{-}} x_{P}\left(\sum_{Q: \ell_{Q} \geq(1-\epsilon) L_{\mathrm{av}}} \frac{x_{Q} \ell_{Q}}{n} \cdot \frac{\ell_{Q}-\ell_{P}^{+}}{\ell_{Q}}\right)^{2} \cdot \frac{1}{\sum_{Q: \ell_{Q} \geq(1-\epsilon) L_{\mathrm{av}} \frac{x_{Q} \ell_{Q}}{n}}} \\
& =-\frac{\lambda}{d} \sum_{P \in \mathcal{P}_{\epsilon, \nu}^{-}} x_{P}\left(\sum_{Q: \ell_{Q} \geq(1-\epsilon) L_{\mathrm{av}}} \frac{x_{Q}}{n} \cdot\left(\ell_{Q}-\ell_{P}^{+}\right)\right)^{2} \cdot \frac{1}{C T} \\
& =-\frac{\lambda}{d} \sum_{P \in \mathcal{P}_{\epsilon, \nu}^{-}} x_{P}\left(T \cdot\left(C-\ell_{P}^{+}\right)\right)^{2} \cdot \frac{1}{C T} \\
& \leq-\frac{\lambda}{d}\left(T \cdot\left(C-(1-\epsilon) L_{\mathrm{av}}\right)\right)^{2} \cdot \frac{1}{C T} \cdot \sum_{P \in \mathcal{P}_{\epsilon, \nu}^{-}} x_{P} .
\end{aligned}
$$

Finally, using Equation (8) and $C T \leq L_{\mathrm{av}}$,

$$
\begin{aligned}
\mathbb{E}\left[\sum_{P, Q} V_{P Q}\right] & \leq-\frac{\lambda}{d}\left(\epsilon L_{\mathrm{av}}\right)^{2} \cdot \frac{1}{C T} \cdot \sum_{P \in \mathcal{P}_{\epsilon, \nu}^{-}} x_{P} \\
& \leq-\frac{\lambda \epsilon^{2} L_{\mathrm{av}}}{d} \delta n \\
& \leq-\Omega\left(\frac{\delta \epsilon^{2} \Phi}{d}\right) .
\end{aligned}
$$

In both cases, the potential decreases by at least a factor of $\left(1-\Omega\left(\epsilon^{2} \delta / d\right)\right)$ in expectation, which, by Lemma 20, implies that the expected time to reach a state with $\Phi(x(t)) \leq \Phi^{*}$ is at most the time stated in the theorem.

From Theorem 7 we can immediately derive the next corollary.

Corollary 8. Consider a symmetric network congestion game with polynomial latency functions of maximum degree $d$ and with minimum and maximum coefficients $a_{\max }$ and $a_{\min }$, respectively. If all players use the IMITATION PROTOCOL, then the expected convergence time of imitation dynamics to an $(\delta, \epsilon, \nu)$-equilibrium is upper bounded by

$$
\mathcal{O}\left(\frac{d^{2}}{\epsilon^{2} \delta} \cdot \log \left(n m \frac{a_{\max }}{a_{\min }}\right)\right) .
$$

Let us remark, that $(\delta, \epsilon, \nu)$-equilibria are transient, i. e., they can be left again once they are reached, for example, if the average latency decreases or if agents migrate towards low-latency paths. However, our proofs actually do not only bound the time until a $(\delta, \epsilon, \nu)$-equilibrium is reached for the first time, but rather the expected total number of rounds in which the system is not at a $(\delta, \epsilon, \nu)$-equilibrium.

Note that in the definition of $(\delta, \epsilon, \nu)$-equilibria we require the majority of agents to deviate by no more than a small amount from $L_{\mathrm{av}}^{+}$. This is because the expected latency of a path sampled by an agent is $L_{\mathrm{av}}$, but the latency of the destination path becomes larger if the agent migrates. We use $L_{\mathrm{av}}^{+}$as an upper bound in our proof, although we could use a slightly smaller quantity in cases where the origin $Q$ and the destination $P$ intersect, namely $\ell_{P}\left(x+1_{P}-1_{Q}\right)$. Using an average over $P$ and $Q$ of this quantity rather than $L_{\text {av }}^{+}$would result in a slightly stronger definition of $(\delta, \epsilon, \nu)$-equilibria. However, we go with the definition as presented above for the sake of clarity of presentation. 
Let us conclude this section by showing that there are fundamental limitations to fast convergence. One could hope to show fast convergence towards a state in which all agents are approximately satisfied, i.e., $\delta=0$. However, any protocol that proceeds by sampling either a strategy or an agent and then possibly migrates, takes at least expected time $\Omega(n)$ to reach a state in which all agents sustain a latency that is within a constant factor of $L_{\mathrm{av}}^{+}$. To see this, consider an instance with $n=2 \mathrm{~m}$ agents and identical linear latency functions. Now, let $x_{1}=3, x_{2}=1$ and $x_{i}=2$ for $3 \leq i \leq n$. Then, the probability that one of the players currently using resource 1 samples resource 2 is at most $\mathcal{O}(1 / m)=\mathcal{O}(1 / n)$. Since this is the only possible improvement step, this yields the desired bound.

\section{Imitation Dynamics in Singleton Games}

In this section, we improve on our previous results and consider imitation dynamics in the special case of singleton congestion games. A major drawback of the IMITATION PROTOCOL is that players who rely on this protocol cannot explore the complete set of edge if the dynamics start in a state in which some edges are unused. Even worse, the event that an edge becomes unused in later states, although it has been used in the initial state, is not impossible. It is clear, however, that when starting from a random initial distribution of players among the edges, the probability of emptying an edge becomes increasingly unlikely as the number of players increases.

Subsequently, we formalize this statement in the following sense. Consider a family of singleton congestion games over the same set of edges with latency functions without offsets. Then, the probability that an edge becomes unused is exponentially small in the number of players. To this end, consider a vector of continuous latency functions $\mathcal{L}=\left(\ell_{e}\right)_{i \in[m]}$ with $\ell_{e}:[0,1] \rightarrow \mathbb{R}_{>0}$. To use these functions for games with a finite number of players, we have to normalize them appropriately. For any such function $\ell \in \mathcal{L}$, let $\ell^{n}$ with $\ell^{n}(x)=\ell(x / n)$ denotes the respective scaled function. We may think of this as having $n$ agents with weight $1 / n$ each. Note that this transformation leaves the elasticity unchanged, whereas the step size $\nu$ decreases as $n$ increases. For a vector of latency functions $\mathcal{L}=\left(\ell_{e}\right)_{i \in[m]}$, let $\mathcal{L}^{n}=\left(\ell_{e}^{n}\right)_{i \in[m]}$.

Theorem 9. Fix a vector of latency functions $\mathcal{L}$ with $\ell_{e}(0)=0$ for all $i \in[m]$. For the singleton congestion game over $\mathcal{L}^{n}$ with $n$ players, the probability that the IMITATION PROTOCOL with random initialization generates a state with $x_{e}=0$ for some $i \in[m]$ within poly $(n)$ rounds is bounded by $2^{-\Omega(n)}$.

Proof. Let $d$ denote an upper bound on the elasticity of the functions in $\mathcal{L}$, and let opt $\mathcal{L}_{\mathcal{L}}=\min _{y}\left\{L_{\text {av }}(y)\right\}$ where the minimum is taken over all $y \in\left\{y^{\prime} \in \mathbb{R}_{\geq 0}^{m} \mid \sum_{e} y_{e}^{\prime}=1\right\}$. In other words, opt ${ }_{\mathcal{L}}$ corresponds to the minimum average latency achievable in a fractional solution. For any $e \in[m]$, by continuity and monotonicity, there exists an $y_{e}>0$ such that $\ell_{e}\left(y_{e}\right)<\operatorname{opt}_{\mathcal{L}} / 4^{d}$ and $y_{e}<1 / \mathrm{m}$.

Consider the congestion game with $n$ players and fix an arbitrary edge $e \in[m]$. In the following, we upper bound the probability that the congestion on edge $e$ falls below $n y_{e} / 2$. First, consider the random initialization in which each resource receives an expected number of $n / m$ agents. The probability that $x_{e}<$ $n y_{e} / 2 \leq n /(2 m)$ is at most $2^{-\Omega\left(n y_{e}\right)}$. Now, consider any assignment $x$ with $x_{j}>n y_{j} / 2$ for all $e \in[m]$. There are two cases.

Case 1: $x_{e}>y_{e} n$. Since in expectation, our policy removes at most a $\lambda / d$ fraction of the agents from edge $e$, the expected load in the subsequent round is at least $(1-\lambda / d) x_{e}$. Since for sufficiently small $\lambda$ it holds that $1-\lambda / d \geq 3 / 4$, we can apply a Chernoff bound (Fact 16) in order to obtain an upper bound of $2^{-\Omega\left(x_{e}\right)}$ for the probability that the congestion on $e$ decreases to below $x_{e} / 2 \geq y_{e} n / 2$.

Case 2: $y_{e} n / 2<x_{e} \leq y_{e} n$. Hence, $\ell_{e}^{n}\left(x_{e}\right) \leq \operatorname{opt}_{\mathcal{L}} / 4^{d}$. In the following, let $n^{-}$denote the number of agents on edges $r$ with $\ell_{r}^{n}\left(x_{r}+1\right)<\ell_{e}^{n}\left(x_{e}\right)$, and let $n^{+}$denote the number of players utilizing edges with latency above opt ${ }_{\mathcal{L}}$. There are two subcases:

Case 2a: $n^{-}=0$. Then, the probability that an agent leaves edge $e$ is 0 . 
Case 2b: $n^{-} \geq 1$. We first show that $n^{+} \geq 4 \max \left\{n^{-}, x_{e}\right\}$. For the sake of contradiction, assume that $n^{+}<4 n^{-}$. Now, consider an assignment where all of these players are shifted to edges $r$ with latency $\ell_{r}^{n}\left(x_{r}\right)<\ell_{e}^{n}\left(x_{e}\right) \leq \operatorname{opt}_{\mathcal{L}} / 4^{d}$, where edge $r$ receives $n^{+} \cdot x_{r} / n^{-}$(fractional) players. In this assignment, the congestion on all edges is increased by no more than a factor of $n^{+} / n^{-}<4$. Hence, due to the limited elasticity, this increases the latency by strictly less than a factor of $4^{d}$. Then, all edges have a latency of less than opt ${ }_{\mathcal{L}} / 4 \cdot 4=\operatorname{opt}_{L}$ and some have latency strictly less than $\mathrm{opt}_{L}$, a contradiction. The same argument also holds if we consider only resource $e$ rather than all resources $r$ considered above. Hence, also $n^{+} \geq 4 x_{e}$.

Now, consider the number of players leaving edge $e$. Clearly,

$$
\mathbb{E}\left[\Delta X_{e}^{-}\right] \leq x_{e} \cdot \frac{\lambda}{d} \sum_{r: \ell_{r}^{n}\left(x_{r}+1\right)<\ell_{e}^{n}\left(x_{e}\right)} \frac{x_{r}}{n}=x_{e} \cdot \frac{\lambda n^{-}}{d n} .
$$

All players with current latency at least opt ${ }_{\mathcal{L}}$ can migrate to resource $e$ since the anticipated latency gain is larger than $\nu$. Hence, the number of players migrating towards $e$, is at least

$$
\begin{aligned}
\mathbb{E}\left[\Delta X_{e}^{+}\right] & \geq \sum_{r: \ell_{r}^{n}\left(x_{r}\right) \geq \mathrm{opt}_{\mathcal{L}}} x_{r} \cdot \frac{\lambda x_{e} \cdot\left(\ell_{r}^{n}\left(x_{r}\right)-\ell_{e}^{n}\left(x_{e}+1\right)\right)}{n d \ell_{r}^{n}\left(x_{r}\right)} \\
& \geq \frac{\lambda x_{e}}{n d} \cdot \sum_{r: \ell_{r}^{n}\left(x_{r}\right) \geq \mathrm{opt}_{\mathcal{L}}} x_{r} \cdot \frac{\ell_{r}^{n}\left(x_{r}\right)-2^{d} \cdot \ell_{e}^{n}\left(x_{e}\right)}{\ell_{r}^{n}\left(x_{r}\right)} \\
& \geq \frac{\lambda x_{e}}{n d} \cdot\left(1-\frac{1}{2^{d}}\right) \cdot n^{+} \\
& \geq 2 \cdot x_{e} \cdot \frac{\lambda}{d n} \max \left\{n^{-}, x_{e}\right\} .
\end{aligned}
$$

The third inequality holds since $\ell_{r}^{n} \geq \operatorname{opt}_{\mathcal{L}}$ and $\ell_{e}^{n} \leq \operatorname{opt}_{\mathcal{L}} / 4^{d}$ and the last inequality holds since $d \geq 1$. For any $T \geq 0$ it holds that

$$
\begin{aligned}
\mathbb{P}\left[\Delta X_{e} \geq 0\right] & \geq \mathbb{P}\left[\left(\Delta X_{e}^{+} \geq T\right) \wedge\left(\Delta X_{e}^{-} \leq T\right)\right] \\
& \geq\left(1-\mathbb{P}\left[\Delta X_{e}^{+}<T\right]\right) \cdot\left(1-\mathbb{P}\left[\Delta X_{e}^{-}>T\right]\right) .
\end{aligned}
$$

Due to our lower bounds on $\mathbb{E}\left[\Delta X_{e}^{+}\right]$and $\mathbb{E}\left[\Delta X_{e}^{-}\right]$we can apply a Chernoff bound (Fact 16) on these probabilities. We set $T=1.5 \lambda \max \left\{x_{e}, n^{-}\right\} x_{e} /(d n)$ which is an upper bound on $\mathbb{E}\left[\Delta X_{e}^{-}\right]$and a lower bound on $\mathbb{E}\left[\Delta X_{e}^{+}\right]$, so

$$
\begin{aligned}
& \mathbb{P}\left[\Delta X_{e}^{+}<T\right] \leq 2^{-\Omega(T)} \leq 2^{-\Omega\left(\lambda x_{e}^{2} /(d n)\right)} \text { and } \\
& \mathbb{P}\left[\Delta X_{e}^{-}>T\right] \leq 2^{-\Omega(T)} \leq 2^{-\Omega\left(\lambda x_{e}^{2} /(d n)\right)} .
\end{aligned}
$$

Altogether,

$$
\begin{aligned}
\mathbb{P}\left[\Delta X_{e} \geq 0\right] & \geq\left(1-2^{-\Omega\left(\frac{\lambda x_{e}^{2}}{d n}\right)}\right) \cdot\left(1-2^{-\Omega\left(\frac{\lambda x_{e}^{2}}{d n}\right)}\right) \\
& =1-2^{-\Omega\left(\frac{\lambda x_{e}^{2}}{d n}\right)} .
\end{aligned}
$$

Finally, since $x_{e} \geq n y_{e} / 2, \mathbb{P}\left[\Delta X_{e}<0\right] \leq 2^{-\Omega\left(\lambda n y_{e}^{2} / d\right)}=2^{-\Omega\left(x_{e}\right)}$.

In all cases, the probability that the edge becomes unused is bounded by $2^{-\Omega\left(x_{e}\right)}=2^{-\Omega(n)}$. Hence, the same holds also for $m=\operatorname{poly}(n)$ edges and $\operatorname{poly}(n)$ rounds.

The proof does not only show that edges do not become empty with high probability, but also that the congestion does not fall below any constant congestion value. In particular, for the constant $d$ this implies that with high probability the dynamics never reach case $2 b$ of the proof of Lemma 2 . This is the only place where our analysis relies on the parameter $\nu$. Hence, for a large number of players we can remove it from the protocol and the dynamics converge to an exact Nash equilibrium. 


\subsection{The Price of Imitation}

In the preceding section we have seen that it is unlikely that resources become unused when the granularity of an agent decreases. If the instance, i. e., the latency functions and the number of users, is fixed, it is an interesting question, how much the performance can suffer from the fact that the IMITATION PROTOCOL is not innovative. We measure this degradation of performance by introducing the Price of Imitation which is defined as the ratio between the expected social cost of the state to which the IMITATION PROTOCOL converges, denoted $I_{\Gamma}$, and the optimum social cost. The expectation is taken over the random choices of the IMITATION PROTOCOL, including random initialization.

We answer this question here for the case of linear latency functions of the form $\ell_{e}(x)=a_{e} x$. Then, $d=1$ is an upper bound on the elasticity and $\nu=a_{\max }=\max _{e \in E}\left\{a_{e}\right\}$. Choosing the average latency $S C(x)=\sum_{e \in E}\left(x_{e} / n\right) \cdot \ell_{e}\left(x_{e}\right)$ as the social cost measure, we show that the Price of Imitation is bounded by a constant. It is, however, obvious that the same also holds if we consider the makespan, i. e., the maximum latency, as social cost function.

The performance of the dynamics can be artificially degraded by introducing an extremely slow edge. Thus, $a_{\max }$ can be chosen extremely large such that any state is imitation-stable. However, such a resource can be removed from the instance without harming the optimal solution at all since it would not be used anyhow. We will call such resources useless and make this notion precise below.

Let us first define some quantities used in the proof. For a set of resources $M$, let $A_{M}=\sum_{e \in M} \frac{1}{a_{e}}$ and let $A_{\Gamma}=A_{[m]}$. For $M \subseteq[m]$ let $\Gamma \backslash M$ denote the instance obtained from $\Gamma$ by removing all resources in $M$. In the proof, we do not compare the outcome of the IMITATION PROTOCOL to the optimum solution, but rather to a lower bound, namely the optimal fractional solution. The optimal fractional solution $\tilde{x}_{e}$ can be computed as $\tilde{x}_{e}=n /\left(A_{\Gamma} a_{e}\right)$. For this solution, the latency of all resources is $a_{e} \cdot \tilde{x}_{e}=n / A_{\Gamma}$. A resource is useless if $\tilde{x}_{e}<1$. In the following, we assume that there are no useless resources. Then, we can show that the social cost at an imitation-stable state in which all resources are used, does not differ by more than a small constant from the optimal social cost (Lemma 11) and that the Price of Imitation is small. In fact, whereas $\tilde{x}_{e} \geq 1$ is required for Lemma 11 , we here need a slightly stronger assumption, namely that $x_{e}=\Omega(\log n)$.

Theorem 10. Assume that for the optimal fractional solution, $\tilde{x}_{e}=\Omega(\log n)$ large enough. The price of imitation is at most $(3+o(1))$. In particular, for $\delta>0$, and any $n \geq n_{0}(\delta)$ for a large enough value $n_{0}(\delta)$ (which is independent of the instance),

$$
I_{\Gamma} \leq(3+\delta) \cdot \frac{n}{A_{\Gamma}}
$$

We start by proving two lemmas.

Lemma 11. Let $x$ be a state in which no agent can gain more than $a_{\max }$ Then,

$$
\frac{n}{A_{\Gamma}} \leq S C(x) \leq 3 \frac{n}{A_{\Gamma}}
$$

Proof. The lower bound has been proven above since $n / A_{\Gamma}$ is the social cost of an optimal fractional solution. Also note that, since there are no useless resources, $\tilde{x}_{e} \geq 1$ and hence $n / A_{\Gamma} \geq a_{\max }$.

For the upper bound, consider a state $x$ in which no agent can gain more than $a_{\max }$. For the sake of contradiction assume that there exists a resource $e \in[m]$ with $\ell_{e}\left(x_{e}\right)>3 n / A_{\Gamma}$. Since $x \neq \tilde{x}$ there exists a resource $f \neq e$ with $x_{f}<\tilde{x}_{f}$. In particular, $\ell_{f}\left(x_{f}+1\right)<n / A_{\Gamma}+a_{\max } \leq 2 n / A_{\Gamma} \leq \ell_{e}\left(x_{e}\right)-a_{\max }$. The last inequality holds due to our assumption on $\ell_{e}\left(x_{e}\right)$ and since $n / A_{\Gamma} \geq a_{\max }$. Hence, any agent on resource $e$ can improve by $a_{\text {max }}$ by migrating to $f$, a contradiction.

Lemma 12. The IMITATION PROTOCOL converges towards an imitation-stable state in time $\mathcal{O}\left(n^{4} \log n\right)$. 
Proof. Consider a state $x(t)$ in which there is at least one agent who can make an improvement of $a_{\max }$. Since its current latency is at most $n \cdot a_{\max }$ and the probability to sample the correct resource is at least $1 / n$, the probability to do so is at least $\lambda \cdot(1 / n) \cdot\left(a_{\max } /\left(n a_{\max }\right)\right)=\lambda / n^{2}$ and the virtual potential gain of such a step is $a_{\max } \geq \Phi / n^{2}$. Hence, the expected virtual potential gain in state $x(t)$ is at least $\lambda \Phi(x(t)) / n^{4}$. Hence, by Lemma 2 ,

$$
\mathbb{E}[\Phi(x(t+1))] \leq \Phi(x(t)) \cdot\left(1-\frac{\lambda}{2 n^{4}}\right)
$$

Note that $\Phi^{*} \geq n a_{\min }$ and $a_{\max } \leq n a_{\min }$ by the assumption that no resource is useless. Also, $\Phi(x(0)) \leq$ $n^{2} a_{\text {max }}$. Now, the theorem is an application of Lemma 21 in the appendix.

Based upon the proof of Theorem 9 we can now bound the probability that a resource becomes empty for the case of linear latency functions more specifically.

Lemma 13. The probability that all resources of the subset $M \subseteq[\mathrm{m}]$ become empty in one round simultaneously is bounded from above by

$$
\prod_{e \in M} 2^{-\Omega\left(\frac{n}{A_{\Gamma} a_{e}}\right)} .
$$

Proof. Recall the bounds on the probability that a resource $e \in[m]$ becomes empty in the proof of Theorem 9 . Since we now consider linear latency functions, we may explicitly compute the value of $y_{e}=1 /\left(A_{\Gamma} a_{e}\right)$. Recall the two cases and the failure probability in the initialization:

Initialization: Here, the error probability was at most $2^{-\Omega\left(n y_{e}\right)}=2^{-\Omega\left(\frac{n}{A_{\Gamma} a_{e}}\right)}$.

Case 1: $x_{e}>y_{e} n$. Here, the error probability was at most $2^{-\Omega\left(x_{e}\right)}=2^{-\Omega\left(\frac{n}{A_{\Gamma} a_{e}}\right)}$.

Case 2: $y_{e} n / 2<x_{e} \leq y_{e} n$. Here, the error probability was at most $2^{-\Omega\left(x_{e}^{2} / n\right)}=2^{-\Omega\left(\frac{n}{\left(A_{\Gamma} a_{e}\right)^{2}}\right)}$.

In all cases, the probability that resource $i$ becomes empty is at most $2^{-\Omega\left(\frac{n}{A_{\Gamma} a_{e}}\right)}$.

Furthermore, consider resources $e$ and $e^{\prime}$ and let $E$ and $E^{\prime}$ denote the events that $e$ and $e^{\prime}$ become empty, respectively. It holds that, $\mathbb{P}\left[E^{\prime} \mid E\right] \leq \mathbb{P}\left[E^{\prime}\right]$. Therefore, $\mathbb{P}\left[E \cap E^{\prime}\right]=\mathbb{P}[E] \cdot \mathbb{P}\left[E^{\prime} \mid E\right] \leq \mathbb{P}[E] \cdot \mathbb{P}\left[E^{\prime}\right]$. Extending this argument to several resources yields the statement of the lemma.

Using the above two lemmas, we can now prove the main theorem of this section.

Proof of Theorem 10. The proof is by induction on the number of resources $m$. Clearly, the statement holds for $m=1$, in which case there is only one assignment. In the following we divide the sequence of state generated by the IMITATION PROTOCOL into phases consisting of several rounds. The phase is terminated by one of the following events, whatever happens first:

1. A subset of resources $M$ becomes empty.

2. The Imitation PRotocol reaches an imitation-stable state.

3. The protocol enters round $\Theta\left(n^{5} \log n\right)$.

If a phase ends because Event 1 occurs, we start a new phase for the instance $\Gamma \backslash M$. If it ends because of Event 3, we start a new phase for the original instance.

The probability for Event 1 is bounded by Lemma 13. Note that the probability is also bounded for up to poly $(n)$ many rounds. If a phase ends with Event 2 we have $I_{\Gamma} \leq 3 \frac{n}{A_{\Gamma}}$ (Lemma 11). We bound the probability of this event by 1 , which is trivially true. Event 3 happens with a probability at most $\mathcal{O}(1 / n)$. 
This can be shown using Lemma 12 and Markov's inequality. Note that the expected social cost is still at most $I_{\Gamma}$. Summing up over all three events, we obtain the following recurrence:

$$
I_{\Gamma} \leq \sum_{M \subset[m]} \prod_{e \in M} 2^{-\Omega\left(\frac{n}{A_{\Gamma} a_{e}}\right)} \cdot I_{\Gamma \backslash M}+3 \cdot \frac{n}{A_{\Gamma}}+\mathcal{O}\left(\frac{1}{n}\right) \cdot I_{\Gamma}
$$

implying

$$
I_{\Gamma} \cdot\left(1-\mathcal{O}\left(\frac{1}{n}\right)\right) \leq 3 \cdot \frac{n}{A_{\Gamma}}+\sum_{M \subset[m]} \prod_{e \in M} 2^{-\Omega\left(\frac{n}{A_{\Gamma} a_{e}}\right)} \cdot I_{\Gamma \backslash M} .
$$

Substituting the induction hypothesis for $I_{\Gamma \backslash M}$, and introducing a constant $c$ for the constant in the $\Omega()$,

$$
\begin{aligned}
I_{\Gamma} \cdot\left(1-\mathcal{O}\left(\frac{1}{n}\right)\right) & \leq 3 \cdot \frac{n}{A_{\Gamma}}+\sum_{M \subset[m]} \prod_{e \in M} 2^{-\frac{c n}{A_{\Gamma} a_{e}}} \cdot 4 \frac{n}{A_{\Gamma \backslash M}} \\
& =3 \cdot \frac{n}{A_{\Gamma}}+4 \frac{n}{A_{\Gamma}} \sum_{M \subset[m]} 2^{-\frac{c n A_{M}}{A_{\Gamma}}} \cdot \frac{A_{\Gamma}}{A_{\Gamma \backslash M}} .
\end{aligned}
$$

Now, by our assumption that for all $e \in M, \tilde{x}_{e}=n /\left(A_{\Gamma} \cdot a_{e}\right) \geq \Omega(\log n)$, we know that for all $e, 1 / a_{e} \geq$ $c^{\prime} A_{\Gamma} \cdot \log n / n$ for a constant $c^{\prime}$ which we may choose appropriately. In particular, $A_{M} \geq|M| c^{\prime} A_{\Gamma} \cdot \log n / n$ and $A_{\Gamma \backslash M} \geq c^{\prime} A_{\Gamma} \cdot \log n / n$. Altogether,

$$
\begin{aligned}
I_{\Gamma} \cdot\left(1-\mathcal{O}\left(\frac{1}{n}\right)\right) & \leq \frac{n}{A_{\Gamma}}\left(3+4 \sum_{M \subset[m]} 2^{-c c^{\prime}|M| \log n} \cdot \frac{n}{c^{\prime} \log n}\right) \\
& =\frac{n}{A_{\Gamma}}\left(3+4 \sum_{k=1}^{m-1}\left(\begin{array}{c}
m \\
k
\end{array}\right) 2^{-c c^{\prime} k \log n} \cdot \frac{n}{c^{\prime} \log n}\right) \\
& \leq \frac{n}{A_{\Gamma}}\left(3+4 \sum_{k=1}^{m-1} n^{k} \cdot 2^{-c c^{\prime} k \log n} \cdot \frac{n}{c^{\prime} \log n}\right) \\
& \leq \frac{n}{A_{\Gamma}}\left(3+4 \sum_{k=1}^{m-1} 2^{-\left(c c^{\prime}-1\right) k \log n} \cdot \frac{n}{c^{\prime} \log n}\right) \\
& \leq \frac{n}{A_{\Gamma}}\left(3+4 \sum_{k=1}^{m-1} \frac{n^{-\left(c c^{\prime}-1\right) k+1}}{c^{\prime} \log n}\right) \\
& \leq(3+o(1)) \frac{n}{A_{\Gamma}},
\end{aligned}
$$

since the last sum is bounded by $o(n)$. This implies our claim.

\section{Exploring New Strategies}

In Section 3, we have seen that, in the long run, the dynamics resulting from the IMITATION PROTOCOL converges to an imitation-stable state in pseudopolynomial time. The IMITATION PROTOCOL and the concept of an imitation-stable state have the drawback that the dynamics can stabilize in a quite disadvantageous situation, e.g. when all players play the same expensive strategy. This is due to the fact that the strategy space is essentially restricted to the current strategy choices of the agents. Strategies that might be attractive and offer a large latency gain are "lost" once no player uses them anymore.

A stronger result would be convergence towards a Nash equilibrium. In the literature, several other protocols are discussed. For all of the protocols we are aware of, the probability to migrate from one strategy to 
another depends in some continuous, non-decreasing fashion on the anticipated latency gain, and it becomes zero for zero gain. Hence, in a setting with arbitrary latency functions which we consider here, there always exist simple instances and states that are not at equilibrium and in which only one improvement step is possible which has an arbitrarily small latency gain. Hence, it takes pseudopolynomially long, until an exact Nash equilibrium is reached. Still, it might be desirable to design a protocol which reaches a Nash equilibrium in the long run. There are several ways to achieve this goal. We will discuss three of them here.

Theorem 9 states the following for a particular class of singleton congestion games. With an increasing number of players it becomes increasingly unlikely that useful strategies are lost. This allows to omit the parameter $\nu$ from the protocol. If no strategies are lost for a long period of time, the dynamics will converge towards an exact Nash equilibrium.

Second, we may add an additional "virtual agent" to every strategy, such that the probability to sample a strategy never becomes zero. This has two implications on our analysis. On the one hand, there is a certain base load on all resources, denoted by $x_{e}^{0}$. We then need to have an upper bound on the elasticity of $\ell_{e}\left(x-x_{e}^{0}\right)$ which may be larger than the elasticity of $\ell_{e}(x)$ itself. Furthermore, we have to add $|\mathcal{P}|$ virtual agents, which leaves the analysis of the time of convergence unchanged only if $n=\Omega(|\mathcal{P}|)$.

As a third alternative, we can add an exploration component to the protocol. With a probability of $1 / 2$, the agents can sample another path uniformly at random rather than another agent. In this case, however, the elasticity $d$ cannot be used as a damping factor anymore, since the expected increase of congestion may be much larger than the current load. Rather, we have to reduce the migration probability by a factor $\min \left\{1, \frac{|\mathcal{P}| \ell_{\min }}{\beta n}\right\}$ where $\beta$ is an upper bound on the maximum slope and $\ell_{\min }=\min _{e \in E} \ell_{e}(1)$ is the minimum latency of an empty resource.

Protocol 2 EXPLORATION PROTOCOL, repeatedly executed by all players in parallel.

Let $P$ denote the path of the player in state $x$.

Sample another path $Q \in \mathcal{P}$ uniformly at random.

if $\ell_{P}(x)>\ell_{Q}\left(x+1_{Q}-1_{P}\right)$ then

with probability

$$
\mu_{P Q}=\min \left\{1, \lambda \cdot \frac{|\mathcal{P}| \ell_{\min }}{\beta n} \cdot \frac{\ell_{P}(x)-\ell_{Q}\left(x+1_{Q}-1_{P}\right)}{\ell_{P}(x)}\right\}
$$

migrate from path $P$ to bin $Q$.

end if

Lemma 14. Let $x$ denote a state and let $\Delta x$ denote a random migration vector generated by the EXPLORATION PRotocol. Then,

$$
\mathbb{E}[\Delta \Phi(x, \Delta x)] \leq \frac{1}{2} \sum_{P, Q \in \mathcal{P}} \mathbb{E}\left[V_{P Q}(x, \Delta x)\right]
$$

Proof. Recall that Lemma 1 states the following for every state $x$ and every migration vector $\Delta x$

$$
\Delta \Phi(x, \Delta x) \leq \sum_{P, Q \in \mathcal{P}} V_{P Q}(x, \Delta x)+\sum_{e \in E} F_{e}(x, \Delta x)
$$

Now, in order to proof Lemma 14, we apply the same approach as in the proof of Lemma 2 . Hence, it remains to adapt the upper bound on $\mathbb{E}\left[\Delta \tilde{\ell}_{e}\left(\Delta \tilde{x}_{e}\right)\right]$ to the Exploration Protocol. Note that this is quite simple, 
since due to the linearity of expectation,

$$
\begin{aligned}
\mathbb{E}\left[\Delta \tilde{\ell}_{e}\left(\Delta \tilde{x}_{e}\right)\right] & \leq \beta \mathbb{E}\left[\Delta \tilde{x}_{e}\right] \\
& \leq \beta n \cdot \lambda \cdot \frac{\ell_{\min }|\mathcal{P}|}{\beta n} \cdot \frac{1}{|\mathcal{P}|} \cdot \frac{\ell_{P}-\ell_{Q}^{+}}{\ell_{P}} \\
& \leq \lambda \cdot \frac{\ell_{e}^{+}}{\ell_{Q}^{+}} \cdot\left(\ell_{P}-\ell_{Q}^{+}\right),
\end{aligned}
$$

where we have substituted the migration probability of the protocol and the fact that there are at most $n$ agents that may sample a path containing $e$. This proves Equation (2) if $\lambda$ is chosen small enough. With opposite signs, the same argument holds if $e \in P$.

Since we have omitted the parameter $\nu$ from the protocol, we now need a lower bound on the minimum improvement that is possible when the system is not yet at an imitation-stable state in order to give an upper bound on the convergence time. Formally, let

$$
\kappa=\min _{x} \min _{\substack{P, Q \in \mathcal{P} \\ \ell_{p}(x)>\ell_{Q}\left(x+1_{Q}-1_{P}\right)}}\left\{\ell_{P}(x)-\ell_{Q}\left(x+1_{Q}-1_{P}\right)\right\} .
$$

Theorem 15. Consider a symmetric network congestion game in which all players use the EXPLORATION PROTOCOL. Let $x$ denote the initial state of the dynamics. Then the dynamics converge to a Nash equilibrium in expected time

$$
\mathcal{O}\left(\frac{\Phi(x) \beta n \ell_{\max }}{\ell_{\min } \kappa^{2}}\right)
$$

Proof. In every state which is not a Nash equilibrium there exists an agent currently utilizing path $P \in \mathcal{P}$ and a path $Q \in \mathcal{P}$ such that $\ell_{Q} \leq \ell_{P}-\kappa$. Hence, the expected virtual potential gain is at least

$$
\mathbb{E}\left[V_{P Q}\right] \leq-\frac{1}{|\mathcal{P}|} \cdot \frac{\lambda|\mathcal{P}| \ell_{\min }}{\beta n} \cdot \frac{\kappa}{\ell_{P}} \cdot \kappa \leq-\frac{\lambda \ell_{\min }}{\beta n} \cdot \frac{\kappa^{2}}{\ell_{\max }},
$$

and the true potential gain is at least half of this. Again, Lemma 20 yields the expected time until the potential decreases from at most $\Phi$ to $\Phi^{*} \geq 0$.

It is obvious that an analogue of Lemmas 2 and 14 also holds for any protocol that is a combination of the ImitATION PROTOCOL and the ExPLORATION PROTOCOL, e.g., a protocol in which in every round, every agent executes the one or the other with probability one half. Then, in order to bound the value of $\mathbb{E}\left[\Delta \tilde{\ell}_{e}\left(\Delta \tilde{x}_{e}\right)\right]$, we must make a case differentiation based on whether proportional or uniform sampling dominates the probability that other agents migrate towards resource $e$. Such a protocol combines the advantages of the IMITATION PROTOCOL and the EXPLORATION PROTOCOL: In the long run, it converges to a Nash equilibrium, and reaches an approximate equilibrium as quickly as stated by Theorem 7 (up to a factor of 2).

\section{Conclusion}

We have proposed and analyzed a natural protocol based on imitating profitable strategies for distributed selfish agents in symmetric congestion games. If agents use our IMITATION PROTOCOL, the resulting dynamics converge rapidly to approximate equilibria, in which only a small fraction of players have latency significantly above or below the average. In addition, in finite time the dynamics converges to an imitation-stable state, in which no player can improve its latency by more than $\nu$ by imitating a different player. The IMITATION PROTOCOL and the concept of an imitation-stable state have the drawback that dynamics can stabilize in a 
quite disadvantegous situation, e.g. when all players play the same expensive strategy. This is due to the fact that the strategy space is essentially restricted to the current strategy choices of the agents. Strategies that might be attractive and offer large latency gain are "lost" once no player uses them anymore. For singleton congestion games we showed that this event becomes unlikely to occur as the number of players increases. Then, by removing parameter $\nu$ from the protocol, the dynamics become likely to converge to Nash equilibria. Another approach to avoid losing strategies is to include exploration of the strategy space. Towards this end, we can use an EXPLORATION PROTOCOL, in which players sample from the strategy space directly and then migrate with a certain probability. If every player uses a suitably designed EXPLORATION PROTOCOL (or any random combination of EXPLORATION PROTOCOL and IMITATION PROTOCOL), then the dynamics are always guaranteed to converge to a Nash equilibrium. However, acquiring information about possible strategies and their benefits might be a complex and costly process in practice, and hence such an action should be invoked only rarely. In addition, exploration requires small migration probabilities, because the danger of overshooting is more severe. Thus, on the downside, if the EXPLORATION PROTOCOL is used exclusively, this results in significantly larger convergence times.

\section{References}

[1] Heiner Ackermann, Heiko Röglin, and Berthold Vöcking. On the impact of combinatorial structure on congestion games. In Proc. 47th Annual IEEE Symposium on Foundations of Computer Science (FOCS), pages 613-622, 2006.

[2] Baruch Awerbuch, Yossi Azar, and Amir Epstein. The price of routing unsplittable flow. In Proc. 37th Annual ACM Symposium on Theory of Computing (STOC), pages 57-66, 2005.

[3] Baruch Awerbuch, Yossi Azar, Amir Epstein, Vahab Mirrokni, and Alexander Skopalik. Fast convergence to nearly optimal solutions in potential games. In Proc. 9th ACM Conference on Electronic Commerce, pages 264-273, 2008.

[4] Petra Berenbrink, Tom Friedetzky, Leslie Ann Goldberg, Paul Goldberg, Zengjian Hu, and Russel Martin. Distributed selfish load balancing. In Proc. 17th Annual ACM-SIAM Symposium on Discrete Algorithms (SODA), pages 354-363, 2006.

[5] Petra Berenbrink, Tom Friedetzky, Iman Hajirasouliha, and Zengjian Hu. Convergence to equilibria in distributed, selfish reallocation processes with weighted tasks. In Proc. 15th Annual European Symposium on Algorithms (ESA), pages 41-52, 2007.

[6] Avrim Blum, Eyal Even-Dar, and Katrina Ligett. Routing without regret: On convergence to Nash equilibria of regret-minimizing algorithms in routing games. In Proc. 25th Annual ACM Symposium on Principles of Distributed Computing (PODC), pages 45-52, 2006.

[7] Steve Chien and Alistair Sinclair. Convergence to approximate Nash equilibria in congestion games. In Proc. 18th Annual ACM-SIAM Symposium on Discrete Algorithms (SODA), pages 169-178, 2007.

[8] George Christodoulou and Elias Koutsoupias. The price of anarchy of finite congestion games. In Proc. 37th Annual ACM Symposium on Theory of Computing (STOC), pages 67-73, 2005.

[9] Eyal Even-Dar, Alexander Kesselman, and Yishay Mansour. Convergence time to Nash equilibria. In Proc. 30th International EATCS Colloquium on Automata, Languages and Programming (ICALP), pages 502-513, 2003.

[10] Eyal Even-Dar and Yishay Mansour. Fast convergence of selfish rerouting. In Proc. 16th Annual ACMSIAM Symposium on Discrete Algorithms (SODA), pages 772-781, 2005.

[11] Alex Fabrikant, Christos Papadimitriou, and Kunal Talwar. The complexity of pure Nash equilibria. In Proc. 36th Annual ACM Symposium on Theory of Computing (STOC), pages 604-612, 2004. 
[12] Angelo Fanelli, Michele Flammini, and Luca Moscardelli. The speed of convergence in congestion games under best-response dynamics. In Proc. 35th International EATCS Colloquium on Automata, Languages and Programming (ICALP), pages 796-807, 2008.

[13] Simon Fischer, Petri Mähönen, Marcel Schöngens, and Berthold Vöcking. Load balancing for dynamic spectrum assignment with local information for secondary users. In Proc. IEEE International Dynamic Access Spectrum Access Networks (DySPAN) Symposium, 2008.

[14] Simon Fischer, Lars Olbrich, and Berthold Vöcking. Approximating Wardrop equilibria with finitely many agents. Distributed Computing, 21(2):129-139, 2008. Special Issue DISC 2007.

[15] Simon Fischer, Harald Räcke, and Berthold Vöcking. Fast convergence to Wardrop equilibria by adaptive sampling methods. In Proc. 38th Annual ACM Symposium on Theory of Computing (STOC), pages 653-662, 2006.

[16] Simon Fischer and Berthold Vöcking. Adaptive routing with stale information. Theoretical Computer Science, 2008. Invited paper. To appear.

[17] Dimitris Fotakis, Alexis Kaporis, and Paul Spirakis. Atomic congestion games: Fast, myopic and concurrent. In Proc. 1st Symposium on Algorithmic Game Theory (SAGT), pages 121-132, 2008.

[18] Paul W. Goldberg. Bounds for the convergence rate of randomized local search in a multiplayer load-balancing game. In Proc. 23rd Annual ACM Symposium on Principles of Distributed Computing (PODC), pages 131-140, 2004.

[19] Torben Hagerup and Christine Rüb. A guided tour of Chernoff bounds. Information Processing Letters, 33:305-308, 1990.

[20] Josef Hofbauer and Karl Sigmund. Evolutionary Games and Population Dynamics. Cambridge University Press, 1998.

[21] Samuel Ieong, Robert McGrew, Eugene Nudelman, Yoav Shoham, and Qixiang Sun. Fast and compact: A simple class of congestion games. In Proceedings of the 20th National Conference on Artificial Intelligence (AAAI), pages 489-494, 2005.

[22] Elias Koutsoupias and Christos Papadimitriou. Worst-case equilibria. In Proc. 16th International Symposium on Theoretical Aspects of Computer Science (STACS), pages 404-413. Springer-Verlag, 1999.

[23] Noam Nisan, Tim Roughgarden, Eva Tardos, and Vijay Vazirani, editors. Algorithmic Game Theory. Cambridge University Press, 2007.

[24] Robert W. Rosenthal. A class of games possessing pure-strategy Nash equilibria. International Journal of Game Theory, 2:65-67, 1973.

[25] Tim Roughgarden and Éva Tardos. How bad is selfish routing? Journal of the ACM, 49(2):236-259, 2002.

[26] Alexander Skopalik and Berthold Vöcking. Inapproximability of pure Nash equilibria. In Proc. 40th Annual ACM Symposium on Theory of Computing (STOC), pages 355-364, 2008.

[27] Jörgen W. Weibull. Evolutionary Game Theory. MIT press, 1995. 


\section{A Appendix}

\section{A.1 Useful Facts}

Throughout the technical part of this paper, we will apply the following two Chernoff bounds.

Fact 16 (Chernoff, see [19]). Let $X$ be a sum of Bernoulli variables. Then, $\mathbb{P}[X \geq k \cdot \mathbb{E}[X]] \leq \mathrm{e}^{-\mathbb{E}[X] k \cdot(\ln k-1)}$, and, for $k \geq 4>\mathrm{e}^{4 / 3}, \mathbb{P}[X \geq k \cdot \mathbb{E}[X]] \leq \mathrm{e}^{-\frac{1}{4} \mathbb{E}[X] k \ln k}$. Equivalently, for $k \geq 4 \mathbb{E}[X], \mathbb{P}[X \geq k] \leq$ $\mathrm{e}^{-\frac{1}{4} k \ln (k / \mathbb{E}[X])}$.

The following fact yields a linear approximation of the exponential function.

Fact 17. For any $r>0$ and $x \in[0, r]$, it holds that $\left(\mathrm{e}^{x}-1\right) \leq x \cdot \frac{\mathrm{e}^{r}-1}{r}$.

Proof. The function $\exp (x)-1$ is convex and it goes through the points $(0,0)$ and $\left(r, \mathrm{e}^{r}-1\right)$, as does the function $x \cdot \frac{\mathrm{e}^{r}-1}{r}$.

Fact 18. For every $c \in] 0,1[$ it holds

$$
\begin{aligned}
\sum_{k=0}^{\infty} c^{k} & =\frac{c}{1-c} \\
\sum_{k=l}^{\infty} c^{k} & =\frac{c^{l}}{1-c}
\end{aligned}
$$

Fact 19 (Jensen's Inequality). Let $f: \mathbb{R} \rightarrow \mathbb{R}$ be a convex function, and let $a_{1}, \ldots, a_{k}, x_{1}, \ldots, x_{k} \in \mathbb{R}$. Then

$$
f\left(\frac{\sum_{i=1}^{k} a_{i} x_{i}}{\sum_{i=1}^{k} a_{i}}\right) \leq \frac{\sum_{i=1}^{k} a_{i} f\left(x_{i}\right)}{\sum_{i=1}^{k} a_{i}} .
$$

If $f(x)=x^{2}$, then

$$
\begin{aligned}
\left(\frac{\sum_{i=1}^{k} a_{i} x_{i}}{\sum_{i=1}^{k} a_{i}}\right)^{2} & \leq \frac{\sum_{i=1}^{k} a_{i}\left(x_{i}\right)^{2}}{\sum_{i=1}^{k} a_{i}} \\
\Leftrightarrow \frac{1}{\sum_{i=1}^{k} a_{i}} \cdot\left(\sum_{i=1}^{k} a_{i} x_{i}\right)^{2} & \leq \sum_{i=1}^{k} a_{i} f\left(x_{i}\right) .
\end{aligned}
$$

Lemma 20 ([14]). Let $X_{0}, X_{1}, \ldots$ denote a sequence of non-negative random variables and assume that for all $i \geq 0$

$$
\mathbb{E}\left[X_{i} \mid X_{i-1}=x_{i-1}\right] \leq x_{i-1}-1
$$

and let $\tau$ denote the first time $t$ such that $X_{t}=0$. Then,

$$
\mathbb{E}\left[\tau \mid X_{0}=x_{0}\right] \leq x_{0}
$$

Lemma 21 ([14]). Let $X_{0}, X_{1}, \ldots$ denote a sequence of non-negative random variables and assume that for all $i \geq 0 \mathbb{E}\left[X_{i} \mid X_{i-1}=x_{i-1}\right] \leq x_{i-1} \cdot \alpha$ for some constant $\alpha \in(0,1)$. Furthermore, fix some constant $x^{*} \in\left(0, x_{0}\right]$ and let $\tau$ be the random variable that describes the smallest $t$ such that $X_{t} \leq x^{*}$. Then,

$$
\mathbb{E}\left[\tau \mid X_{0}=x_{0}\right] \leq \frac{2}{\log (1 / \alpha)} \cdot \log \left(\frac{x_{0}}{x^{*}}\right) .
$$

Again, as a consequence of Lemma 20 the expected time until the potential decreases from at most $\Phi$ to $\Phi$ can be found in the appendix, and which is proved, e. g., in [14]. 